\title{
R-parity violation effect on the top-quark pair production at linear colliders *
}

\author{
Wang Lei ${ }^{2}$, Ma Wen-Gan ${ }^{1,2}$, Hou Hong-Sheng ${ }^{2}$, Zhang Ren-You², and Sun Yan-Bin ${ }^{2}$ \\ ${ }^{1}$ CCAST (World Laboratory), P.O.Box 8730, Beijing 100080, P.R.China \\ ${ }^{2}$ Department of Modern Physics, University of Science and Technology \\ of China (USTC), Hefei, Anhui 230027, P.R.China
}

\begin{abstract}
We investigate in detail the effects of the R-parity lepton number violation in the minimal supersymmetric standard model (MSSM) on the top-quark pair production via both $e^{-}-e^{+}$ and $\gamma-\gamma$ collision modes at the linear colliders. We find that with the present experimental constrained $R$ parameters, the effect from $R$ interactions on the processes $e^{+} e^{-} \rightarrow t \bar{t}$ and $e^{+} e^{-} \rightarrow \gamma \gamma \rightarrow t \bar{t}$ could be significant and may reach $-30 \%$ and several percent, respectively. Our results show that the $R$ effects are sensitive to the c.m.s. energy and the relevant $R$ parameters. However, they are not sensitive to squark and slepton masses when $m_{\tilde{q}} \geq$ $400 \mathrm{GeV}$ (or $m_{\tilde{l}} \geq 300 \mathrm{GeV}$ ) and are almost independent on the $\tan \beta$.
\end{abstract}

PACS: 12.15.Lk, 13.10.+q, 12.60.-i, 12.60.Jv

\footnotetext{
${ }^{*}$ Supported by National Natural Science Foundation of China.
} 


\section{Introduction}

The new physics beyond the standard model (SM) has been intensively studied over the past years [1]. The minimal supersymmetric standard model (MSSM) is currently the most popular one among the extensions of the SM. The R-parity is defined as

$$
R=(-1)^{2 B+L+2 S}
$$

where B, L and S represent the baryon number, lepton number and intrinsic spin of the particle, respectively. This leads to a discrete $Z_{2}$ symmetry in the MSSM lagrangian. For all the SM particles they have $R=1$ and for all of their supersymmetric partners $R=-1$. Usually we consider the R-parity is conserved in the MSSM, but the most general superpotential consistent with the gauge symmetry of the SM can introduce R-parity violating terms as follow [2]

$$
W_{\not k}=\epsilon_{i j}\left(\lambda_{I J K} \tilde{L}_{i}^{I} \tilde{L}_{j}^{J} \tilde{R}^{K}+\lambda_{I J K}^{\prime} \tilde{L}_{i}^{I} \tilde{Q}_{j}^{J} \tilde{D}^{K}+\epsilon_{I} H_{i}^{2} \tilde{L}_{j}^{I}\right)+\lambda_{I J K}^{\prime \prime} \tilde{U}^{I} \tilde{D}^{J} \tilde{D}^{K}
$$

where $\tilde{L}^{I}, \tilde{Q}^{I}, H^{I}$ represent the $\mathrm{SU}(2)$ doublets of lepton, quark and Higgs superfields, respectively, while $\tilde{R}^{I}, \tilde{U}^{I} \tilde{D}^{I}$ are singlets of lepton and quark superfields, I,J,K are flavor indices. All these terms can lead to catastrophically high decay rate for proton. We must require typically $\lambda^{\prime} \lambda^{\prime \prime} \leq 2 \times 10^{-26}\left[\underline{3}\right.$ in order to get a proton lifetime longer than $10^{40} \mathrm{~s}$ [4]. This is highly

unnatural, unless either one or both of $\lambda^{\prime}$ and $\lambda^{\prime \prime}$ are identically zero. In usual MSSM, we require $\mathrm{R}$-parity conservation (i.e. $\lambda^{\prime}=\lambda^{\prime \prime}=0$ ), it constrains the model more than what is really necessary. $\lambda^{\prime}=0$ or $\lambda^{\prime \prime}=0$ is quite adequate. In most models motivated by unification (including supergravity), there is a preference for allowing the lepton number violation over 
the baryon number violation. In addition, the lowest generation $\tilde{U}^{I} \tilde{D}^{J} \tilde{D}^{K}$ operators have the strictest laboratory bounds, e.g. $\lambda_{121}^{\prime \prime} \leq 10^{-6}[\underline{5}$. Because the quark mixing is not zero, it is hard to construct models which allow for large coupling $\lambda^{\prime \prime}$ and satisfy the strict constraint on $\lambda_{121}^{\prime \prime}$. During the last few years experimental search for the effects of $R$ interactions has been done with many efforts. Up to now we have only some upper limits on these $R$ parameters. It is necessary to continue these works on finding $R$ signal or getting further stringent constraints on the $R$ parameters in future experiments.

Top quark was firstly discovered in 1995 by the CDF and D0 at the Fermilab Tevatron. People believe that accurate measurement of top quark pair production at the present and future colliders should be possible in finding the physical effects beyond the SM. Any deviation of observable in top quark pair production process from the SM prediction would give a hint of new physics. Therefore, testing the $R$ effect on top pair production process is an attractive task in high energy experiment.

In previous studies, a lot of effort has been invested in the top quark pair production at present and future colliders, such as the CERN LEP2, CERN Large Hadron Collider (LHC), Tevatron, and the proposed linear colliders (LC): NLC[6], JLC [], TESLA [8] and CLIC [9]. A electron-positron LC can be designed to operate in either $e^{+} e^{-}$or $\gamma \gamma$ collision mode. $\gamma \gamma$ collision is achieved by using Compton backscattered photons in the scattering of intense laser photons on the initial polarized $e^{+} e^{-}$beams [10. At these machines in both $e^{+} e^{-}$and $\gamma \gamma$ collision modes, a great number of top quark pairs can be produced [1]. The signature of this kind of event is much cleaner than that produced at hadron colliders. Ref. 12] presents the calculation of the MSSM 
one-loop radiative corrections to process $e^{+} e^{-} \rightarrow t \bar{t}$ and shows the relative difference between the predictions of the MSSM and the SM is typically below 10\%. Ref. [13] presents the effects of lepton number violating interactions on top-quark pair production via $e^{+} e^{-}$collisions. The NLO QCD corrections in the SM and MSSM to $\gamma \gamma \rightarrow t \bar{t}$ have been discussed in detail in Ref. [14], the corrections are about $10 \%$ and of the order $10^{-2}$, respectively. Ref. [15] demonstrates the SM electroweak $(\mathrm{EW})$ corrections to $\gamma \gamma \rightarrow t \bar{t}$ can reach almost $10 \%$ in the collision energy region

close to the threshold. Ref. [16] gives the $\mathrm{O}\left(\alpha m_{t}^{2} / m_{w}^{2}\right)$ Yukawa corrections to the $e^{+} e^{-} \rightarrow \gamma \gamma \rightarrow t \bar{t}$ in the SM, the general two-Higgs-doublet model (2HDM) as well as the MSSM. The corrections are about a few percent in the SM but can be bigger than $10 \%$ in the MSSM. In Ref. 17] the SUSY EW-like corrections in the $R$-conserving MSSM to $\gamma \gamma \rightarrow t \bar{t}$ are calculated, and the corrections are about a few percent for $\gamma \gamma \rightarrow t \bar{t}$ and one percent for $e^{+} e^{-} \rightarrow \gamma \gamma \rightarrow t \bar{t}$. In this paper, we study the $R$ lepton number violating effects on both the processes $e^{+} e^{-} \rightarrow t \bar{t}$ and $e^{+} e^{-} \rightarrow \gamma \gamma \rightarrow t \bar{t}$ at a LC. The paper is organized as follows. In Sec.II. we give the relevant theory and Feynman diagrams. In Sec.III. we present the analytical calculations. The numerical results and discussions are described in Sec.IV. Finally, we give a short summary. In the Appendix, the related $R$ lepton number violating Feynman rules are listed.

\section{The Relevant Theory and Feynman Diagrams}

In this section we briefly review the theory of MSSM with $R$ lepton number violation. The most general form of the superpotential in the MSSM can be written as [18]:

$$
\mathcal{W}=\mathcal{W}_{M S S M}+\mathcal{W}_{k}
$$


where $\mathcal{W}_{M S S M}$ represents the R-parity conserved term, which can be written as $\mathcal{W}_{M S S M}=\mu \epsilon_{i j} H_{i}^{1} H_{j}^{2}+\epsilon_{i j} l_{I} H_{i}^{1} \tilde{L}_{j}^{I} \tilde{R}^{I}-u_{I}\left(H_{1}^{2} C^{J I *} \tilde{Q}_{2}^{J}-H_{2}^{2} \tilde{Q}_{1}^{J}\right) \tilde{U}^{I}-d_{I}\left(H_{1}^{1} \tilde{Q}_{2}^{I}-H_{2}^{1} C^{I J} \tilde{Q}_{1}^{J}\right) \tilde{D}^{I}$.

The $R$ superpotential part $\mathcal{W}_{k}$ is shown in Eq.(1.2). The soft breaking terms can be expressed as

$$
\begin{aligned}
\mathcal{L}_{\text {soft }}= & -m_{H^{1}}^{2} H_{i}^{1 *} H_{i}^{1}-m_{H^{2}}^{2} H_{i}^{2 *} H_{i}^{2}-m_{L^{I}}^{2} \tilde{L}_{i}^{I *} \tilde{L}_{i}^{I}-m_{R^{I}}^{2} \tilde{R}^{I *} \tilde{R}^{I}-m_{Q^{I}}^{2} \tilde{Q}_{i}^{I *} \tilde{Q}_{i}^{I} \\
& -m_{D^{I}}^{2} \tilde{D}^{I *} \tilde{D}^{I}-m_{U^{I}}^{2} \tilde{U}^{I *} \tilde{U}^{I}+\left(m_{1} \lambda_{B} \lambda_{B}+m_{2} \lambda_{A}^{i} \lambda_{A}^{i}+m_{3} \lambda_{G}^{a} \lambda_{G}^{a}+h . c .\right) \\
& +\left\{B \mu \epsilon_{i j} H_{i}^{1} H_{j}^{2}+B_{I} \epsilon_{I} \epsilon_{i j} H_{i}^{2} \tilde{L}_{j}^{I}+\epsilon_{i j} l_{s I} H_{i}^{1} \tilde{L}_{j}^{I} \tilde{R}^{I}\right. \\
& +d_{s I}\left(-H_{1}^{1} \tilde{Q}_{2}^{I}+C^{I K} H_{2}^{1} \tilde{Q}_{1}^{K}\right) \tilde{D}^{I}+u_{s I}\left(-C^{K I *} H_{1}^{2} \tilde{Q}_{2}^{I}+H_{2}^{2} \tilde{Q}_{1}^{I}\right) \tilde{U}^{I} \\
& +\epsilon_{i j} \lambda_{I J K}^{S} \tilde{L}_{i}^{I} \tilde{L}_{j}^{J} \tilde{R}^{K}+\lambda_{I J K}^{S^{\prime}}\left(\tilde{L}_{i}^{I} \tilde{Q}_{2}^{J} \delta^{J K}-\tilde{L}_{2}^{I} C^{J K} \tilde{Q}_{1}^{J}\right) \tilde{D}^{K}+\lambda_{I J K}^{S^{\prime \prime}} \tilde{U}^{I} \tilde{D}^{J} \tilde{D}^{K} \\
& + \text { h.c. }\} .
\end{aligned}
$$

The bilinear term $\epsilon_{i j} \epsilon_{I} H_{i}^{2} \tilde{L}_{j}^{I}$ is usually considered to be smaller than trilinear terms, so we assume that they are negligible in our work . For the reason mentioned in the introduction we only consider the lepton number violation. This means that $\lambda^{\prime \prime}=0$. Then $\mathcal{L}_{\not k}$ can be written as

$$
\begin{aligned}
\mathcal{L}_{k} & =-\lambda_{i j k}\left[\bar{e}_{k} P_{L} \nu_{i} \tilde{e}_{j L}+\bar{\nu}_{i}^{c} P_{L} e_{j} \tilde{e}_{k L}^{*}+\bar{e}_{k} P_{L} e_{j} \tilde{\nu}_{i L}\right] \\
& -\lambda_{i j k}^{\prime}\left[\bar{d}_{k} P_{L} d_{j} \tilde{\nu}_{i L}+\bar{d}_{k} P_{L} \nu_{i} \tilde{d}_{j}+\bar{\nu}_{i L}^{c} P_{L} d_{j} \tilde{d}_{k R}^{*}-\bar{d}_{k} P_{L} e_{i} \tilde{u}_{j L}-\bar{d}_{k} P_{L} u_{j} \tilde{e}_{i L}-\bar{e}_{i}^{c} P_{L} u_{j} \tilde{d}_{k R}^{*}\right] \\
& -\epsilon_{\alpha \beta \gamma} \lambda_{i j k}^{\prime \prime}\left[\bar{d}_{j}^{c \beta} P_{R} d_{k}^{\gamma} \tilde{u}_{i R}^{\alpha}+\bar{u}_{i}^{c \alpha} P_{R} d_{j}^{\beta} \tilde{d}_{k R}^{\gamma}+\bar{u}_{i}^{c \alpha} P_{R} d_{k}^{\gamma} \tilde{d}_{j R}^{\beta}\right]+h . c .
\end{aligned}
$$

There $\alpha, \beta, \gamma$ are color indices of quarks. From the lagrangian we get the relevant Feynman rules which are listed in Appendix. The Feynman diagrams of the process $e^{+} e^{-} \rightarrow t \bar{t}$ are plotted in Fig.1. Fig.1(a-b) are the tree level R-parity conserving and violating diagrams, respectively. Fig.2 shows the Feynman diagrams of subprocess $\gamma \gamma \rightarrow t \bar{t}$. Fig.2(a) is tree level diagram. Fig.2(b-f) are vertex, box and quartic coupling diagrams with $R$ interactions. Since the Rconserving SUSY EW-like one-loop correction diagrams were already presented in Ref.[17], we 
shall not plot them here. In Fig.2 the diagrams which can be obtained by exchanging the initial photons are not shown.

\section{Calculation}

In all our calculations we use the t'Hooft-Feynman gauge. In the loop diagram calculation we adopt the definitions of one-loop integral functions in Ref.[19], and use the dimensional reduction (DR) scheme[20] and on-mass-shell (OMS) scheme[21] to do renormalization. The numerical calculation of the vector and tensor loop integral functions can be traced back to scalar loop integrals as shown in the Ref. [22].

\section{III.1 Calculation of the process $e^{+} e^{-} \rightarrow t \bar{t}$}

We denote the process of $t \bar{t}$ production via $e^{+} e^{-}$collision as

$$
e^{-}\left(p_{1}\right)+e^{+}\left(p_{2}\right) \rightarrow \bar{t}\left(k_{1}\right)+t\left(k_{2}\right)
$$

where $p_{1}, p_{2}, k_{1}$ and $k_{2}$ are the momenta of the incoming and outgoing particles, respectively. The differential Born cross sections in the R-parity conserving MSSM, corresponding to the diagrams Fig.1(a), can be written as

$$
d \sigma_{M S S M}=d P \frac{N_{c}}{4} \sum_{\text {spin }}\left|A_{\gamma}^{(a)}(\hat{s}, \hat{t}, \hat{u})+A_{Z}^{(a)}(\hat{s}, \hat{t}, \hat{u})\right|^{2},
$$

where $N_{c}=3$, the summation is taken over the spin of the initial and final states, and $d P$ denotes the two-particle phase space element. The factor $1 / 4$ results from the average over the spins of the incoming photons. The $A_{\gamma}^{(a)}$ and $A_{Z}^{(a)}$ represent the amplitudes of the photon and Z 
boson exchange diagrams at tree level, respectively. The Mandelstam kinematical variables are defined as

$$
\hat{s}=\left(p_{1}+p_{2}\right)^{2}, \quad \hat{t}=\left(p_{1}-k_{1}\right)^{2}, \quad \hat{u}=\left(p_{1}-k_{2}\right)^{2},
$$

In the MSSM with the $R$ lepton number violation, the tree level differential cross sections can be expressed as

$$
d \hat{\sigma}_{k^{\prime}}(\hat{s}, \hat{t}, \hat{u})=d P \frac{N_{c}}{4} \sum_{\text {spin }}\left|A_{\gamma}^{(a)}(\hat{s}, \hat{t}, \hat{u})+A_{Z}^{(a)}(\hat{s}, \hat{t}, \hat{u})+\sum_{k=1}^{2,3} A_{\tilde{D}_{k}}^{(b)}(\hat{s}, \hat{t}, \hat{u})\right|^{2},
$$

where $A_{\tilde{D}_{k}}^{(b)},(k=1,2,3)$ are the amplitudes corresponding to diagrams in Fig.1(b). We give the explicit expressions of $A_{\gamma}^{(a)}, A_{Z}^{(a)}$ and $A_{\tilde{D}_{k}}^{(b)}$ as below

$$
\begin{aligned}
& A_{\gamma}^{(a)}(\hat{s}, \hat{t}, \hat{u})=Q_{t} e^{2}\left[\bar{u}\left(k_{1}\right) \gamma_{\mu} v\left(k_{2}\right)\right] \frac{-i}{\hat{s}}\left[\bar{v}\left(p_{2}\right) \gamma_{\mu} u\left(p_{1}\right)\right] \\
& A_{Z}^{(a)}(\hat{s}, \hat{t}, \hat{u})=\frac{e^{2}}{s_{w}^{2} c_{w}^{2}}\left[\bar{u}\left(k_{1}\right) \gamma_{\mu}\left(\frac{P_{L}}{2}-\frac{2 s_{w}^{2}}{3}\right) v\left(k_{2}\right)\right] \frac{i}{\hat{s}-m_{Z}^{2}}\left[\bar{v}\left(p_{2}\right) \gamma_{\mu}\left(-\frac{P_{L}}{2}+s_{w}^{2}\right) u\left(p_{1}\right)\right] \\
& A_{\tilde{D}_{k}}^{(b)}(\hat{s}, \hat{t}, \hat{u})=-\left(\lambda_{13 k}^{\prime}\right)^{2} \sum_{j=1}^{2}\left\{\left[\bar{u}\left(k_{2}\right) Z_{D_{k}}^{2 j} P_{L} u\left(p_{1}\right)\right] \frac{i}{\hat{u}-m_{\tilde{D}_{k j}}^{2}}\left[\bar{v}\left(p_{2}\right) Z_{D_{k}}^{2 j} P_{R} v\left(k_{1}\right)\right]\right\},
\end{aligned}
$$

where $Z_{D_{k}}^{i j}$ represents the elements of the matrix used to diagonalize the down-type squark mass matrix, $k$ is the generation index.

\section{III.2 Calculation of the process $e^{+} e^{-} \rightarrow \gamma \gamma \rightarrow t \bar{t}$}

In this subsection we present the calculation of the process $e^{+} e^{-} \rightarrow \gamma \gamma \rightarrow t \bar{t}$. We denote the subprocess as

$$
\gamma\left(p_{1}\right)+\gamma\left(p_{2}\right) \rightarrow \bar{t}\left(k_{1}\right)+t\left(k_{2}\right)
$$

The Lorentz invariant matrix element at tree level for the process $\gamma \gamma \rightarrow t \bar{t}$ can be written as

$$
A_{0}=A_{0}^{(t)}(\hat{s}, \hat{t}, \hat{u})+A_{0}^{(u)}(\hat{s}, \hat{t}, \hat{u})
$$


where

$$
\begin{aligned}
& A_{0}^{(t)}(\hat{s}, \hat{t}, \hat{u})=-\frac{i Q_{t}^{2} e^{2}}{\hat{t}-m_{t}^{2}} \bar{u}\left(k_{1}\right) \gamma_{\mu}\left(m_{t}+\not k_{1}-\not p_{1}\right) \gamma_{\nu} v\left(k_{2}\right) \varepsilon^{\mu}\left(p_{1}\right) \varepsilon^{\nu}\left(p_{2}\right) \\
& A_{0}^{(u)}(\hat{s}, \hat{t}, \hat{u})=-\frac{i Q_{t}^{2} e^{2}}{\hat{u}-m_{t}^{2}} \bar{u}\left(k_{1}\right) \gamma_{\nu}\left(m_{t}+\not k_{1}-\not p_{2}\right) \gamma_{\mu} v\left(k_{2}\right) \varepsilon^{\nu}\left(p_{2}\right) \varepsilon^{\mu}\left(p_{1}\right)
\end{aligned}
$$

The corresponding differential cross section is written as

$$
\frac{d \hat{\sigma}_{0}(\hat{s}, \hat{t}, \hat{u})}{d \hat{t}}=\frac{1}{4} \frac{N_{c}}{16 \pi \hat{s}^{2}} \sum_{\text {spin }}\left|A_{0}\right|^{2},
$$

where the summation is taken over the spin of initial and final states. The SUSY EW-like corrections in the R-parity conserving MSSM to top pair production via photon-photon collisions were calculated in Ref.[17]. In this work we present only one-loop contributions involving $R$ interactions.

The counterterm of top quark waves function $\delta Z_{t}$ is decided by the one-particle irreducible two-point function $i \Gamma\left(p^{2}\right)$ with the on-mass-shell (OMS) condition. The renormalized $R$ part of the top quark two-point function can be defined as

$$
\hat{\Gamma}_{t t}\left(p^{2}\right)=\left(\not p-m_{t}\right)+\left[\not p P_{L} \hat{\Sigma}_{t t}^{L}\left(p^{2}\right)+\not p P_{R} \hat{\Sigma}_{t t}^{R}\left(p^{2}\right)+P_{L} \hat{\Sigma}_{t t}^{S, L}\left(p^{2}\right)+P_{R} \hat{\Sigma}_{t t}^{S, R}\left(p^{2}\right)\right] .
$$

The corresponding $R$ parts of the unrenormalized self-energies are

$$
\begin{gathered}
\Sigma_{t t}^{S, L}\left(p^{2}\right)=0, \quad \sum_{t t}^{S, R}\left(p^{2}\right)=0, \quad \Sigma_{t t}^{R}\left(p^{2}\right)=0 \\
\Sigma_{t t}^{L}\left(p^{2}\right)=\frac{1}{16 \pi^{2}} \sum_{i=1}^{2,3} \sum_{j=1}^{2} \sum_{k=1}^{2,3}\left(\left|V_{\tilde{D}_{i j} E_{k} t}\right|^{2} B_{0}\left[p^{2}, m_{\tilde{D}_{i j}}^{2}, m_{E_{k}}^{2}\right]\right. \\
\left.+\left|V_{\tilde{D}_{i j} E_{k} t}\right|^{2} B_{1}\left[p^{2}, m_{\tilde{D}_{i j}}^{2}, m_{E_{k}}^{2}\right]+\left|V_{\tilde{E}_{k j} D_{i} t}\right|^{2} B_{1}\left[p^{2}, m_{D_{i}}^{2}, m_{\tilde{E}_{k j}}^{2}\right]\right)
\end{gathered}
$$

where $i, k$ are the generation indexes and $j$ is the sparticle index. Using the OMS renormalization conditions [21], we get the renormalization constants as

$$
\delta \Sigma_{t t}\left(p^{2}\right)=C_{L} \not p P_{L}+C_{R} \not p P_{R}-C_{S}^{L} P_{L}-C_{S}^{R} P_{R}
$$


The renormalization constants for the $t-t-\gamma$ vertex $V_{t t \gamma}$ are

$$
\delta V_{\gamma t t}=-i e \gamma^{\mu}\left[C^{L} P_{L}+C^{R} P_{R}\right]
$$

where

$$
\begin{gathered}
C_{L}=\frac{1}{2}\left(\delta Z_{t t}^{L}+\delta Z_{t t}^{L \dagger}\right), \\
C_{R}=\frac{1}{2}\left(\delta Z_{t t}^{R}+\delta Z_{t t}^{R \dagger}\right), \\
C_{S}^{L}=\frac{m_{t}}{2}\left(\delta Z_{t t}^{L}+\delta Z_{t t}^{R \dagger}\right)+\delta m_{t}, \\
C_{S}^{R}=\frac{m_{t}}{2}\left(\delta Z_{t t}^{R}+\delta Z_{t t}^{L \dagger}\right)+\delta m_{t} . \\
\delta m_{t}=\frac{1}{2} \tilde{R} e\left[m_{t} \Sigma_{t t}^{L}\left(m_{t}^{2}\right)+m_{t} \Sigma_{t t}^{R}\left(m_{t}^{2}\right)+\Sigma_{t t}^{S, L}\left(m_{t}^{2}\right)+\Sigma_{t t}^{S, R}\left(m_{t}^{2}\right)\right] \\
\delta Z_{t t}^{L}=-\tilde{R}_{t t}^{L} \Sigma_{t}^{L}\left(m_{t}^{2}\right)-\frac{1}{m_{t}} \tilde{R} e\left[\Sigma_{t t}^{S, R}\left(m_{t}^{2}\right)-\Sigma_{t t}^{S, L}\left(m_{t}^{2}\right)\right] \\
-m_{t} \frac{\partial}{\partial p^{2}} \tilde{R} e\left\{m_{t} \Sigma_{t t}^{L}\left(p^{2}\right)+m_{t} \Sigma_{t t}^{R}\left(p^{2}\right)\right. \\
\left.+\Sigma_{t t}^{S, L}\left(p^{2}\right)+\Sigma_{t t}^{S, R}\left(p^{2}\right)\right\}\left.\right|_{p^{2}=m_{t}^{2}}, \\
\delta Z_{t t}^{R}=-\tilde{R} e \Sigma_{t t}^{R}\left(m_{t}^{2}\right)-m_{t} \frac{\partial}{\partial p^{2}} \tilde{R} e\left\{m_{t} \Sigma_{t t}^{L}\left(p^{2}\right)+m_{t} \Sigma_{t t}^{R}\left(p^{2}\right)\right. \\
\left.\Sigma_{t t}^{S, L}\left(p^{2}\right)+\Sigma_{t t}^{S, R}\left(p^{2}\right)\right\}\left.\right|_{p^{2}=m_{t}^{2}},
\end{gathered}
$$

where $\tilde{R} e$ takes the real part of the loop integrals. We use $A^{v}, A^{b}, A^{q}, A^{s}$ and $A^{c t}$ to represent the amplitude parts contributed by vertex, box, quartic, self-energy diagrams (shown in Fig.2) and counterterms, respectively. The renormalized matrix elements from the one-loop diagrams 
are written as

$$
\begin{aligned}
\delta A_{1-\text { loop }} & =A^{v}+A^{b}+A^{q}+A^{\text {self }}+A^{\text {ct }} \\
& =\epsilon_{\mu}\left(p_{1}\right) \epsilon_{\nu}\left(p_{2}\right) \bar{u}\left(k_{1}\right)\left\{f_{1} g_{\mu \nu}+f_{2} g_{\mu \nu} \gamma_{5}+f_{3} k_{2 \nu} \gamma_{\mu}+f_{4} g_{\mu \nu} \not_{2}+f_{5} k_{2 \nu} \gamma_{5} \gamma_{\mu}\right. \\
& +f_{6} g_{\mu \nu} \gamma_{5} p_{2}+f_{7} \gamma_{\mu} \gamma_{\nu}+f_{8} k_{2 \nu} \gamma_{\mu} p_{2}+f_{9} \gamma 5 \gamma_{\mu} \gamma_{\nu}+f_{10} k_{2 \nu} \gamma_{5} \gamma_{\mu} p_{2}+f_{11} \gamma_{\mu} \gamma_{\nu} p_{2} \\
& +f_{12} \gamma_{5} \gamma_{\mu} \gamma_{\nu} p_{2}+f_{13} k_{2 \mu} k_{2 \nu}+f_{14} k_{2 \mu} k_{2 \nu} \gamma_{5}+f_{15} k_{2 \mu} \gamma_{\nu}+f_{16} k_{2 \mu} \gamma_{5} \gamma_{\nu} \\
& \left.+f_{17} k_{2 \mu} \gamma_{\nu} p_{2}+f_{18} k_{2 \mu} \gamma_{5} \gamma_{\nu} \not_{2}+f_{19} k_{2 \mu} k_{2 \nu} p_{2}+f_{20} k_{2 \mu} k_{2 \nu} \gamma_{5} p_{2}\right\} v\left(k_{2}\right),
\end{aligned}
$$

where $f_{i}(i=1 \sim 20)$ are the form factors. Then we get the one-loop corrections to the cross section from the $R$ part:

$$
\Delta \hat{\sigma}_{1-\text { loop }}(\hat{s})=\frac{N_{c}}{16 \pi \hat{s}^{2}} \int_{\hat{t}^{-}}^{\hat{t}^{+}} d \hat{t} 2 R e \overline{\sum_{\text {spin }}}\left(A_{0}^{\dagger} \cdot \delta A_{1-\text { loop }}\right)
$$

where $\hat{t}^{ \pm}=\left(m_{t}^{2}-\frac{\hat{s}}{2}\right) \pm \frac{\hat{s}}{2} \sqrt{1-4 m_{t}^{2} / \hat{s}}$, and the bar over the summation means average over initial spins. With the integrated photon luminosity in the $e^{+} e^{-}$collision, the total cross section of the process $e^{+} e^{-} \rightarrow \gamma \gamma \rightarrow t \bar{t}$ can be written as

$$
\hat{\sigma}(s)=\int_{E_{0} / \sqrt{s}}^{x_{\max }} d z \frac{d \mathcal{L}_{\gamma \gamma}}{d z} \hat{\sigma}\left(\gamma \gamma \rightarrow t \bar{t} \quad \text { at } \quad \hat{s}=z^{2} s\right)
$$

with $E_{0}=2 m_{t}$, and $\sqrt{s}(\sqrt{\hat{s}})$ being the $e^{+} e^{-}(\gamma \gamma)$ center-of-mass energy. $\frac{d \mathcal{L}_{\gamma \gamma}}{d z}$ is the distribution function of photon luminosity, which is defined as:

$$
\frac{d \mathcal{L}_{\gamma \gamma}}{d z}=2 z \int_{z^{2} / x_{\max }}^{x_{\max }} \frac{d x}{x} F_{\gamma / e}(x) F_{\gamma / e}\left(z^{2} / x\right)
$$

For the initial unpolarized electrons and laser photon beams, the energy spectrum of the back scattered photon is given by 23$]$

$$
F_{\gamma / e}=\frac{1}{D(\xi)}\left[1-x+\frac{1}{1-x}-\frac{4 x}{\xi(1-x)}+\frac{4 x^{2}}{\xi^{2}(1-x)^{2}}\right]
$$


where

$$
\begin{array}{r}
D(\xi)=\left(1-\frac{4}{\xi}-\frac{8}{\xi^{2}}\right) \ln (1+\xi)+\frac{1}{2}+\frac{8}{\xi}-\frac{1}{2(1+\xi)^{2}}, \\
\xi=\frac{4 E_{0} \omega_{0}}{m_{e}^{2}},
\end{array}
$$

$m_{e}$ and $E_{0}$ are the incident electron mass and energy, respectively, $\omega_{0}$ is the laser-photon energy, and $x$ is the fraction of the energy of the incident electron carried by the backscattered photon. In our calculation, we choose $\omega_{0}$ such that it maximizes the backscattered photon energy without spoiling the luminosity via $e^{+} e^{-}$pair creation. Then we have $\xi=2(1+\sqrt{2}), x_{\max } \simeq 0.83$, and $D(\xi)=1.8$

\section{Numerical results and discussion}

We take the input parameters as $m_{e}=0.511 \mathrm{MeV}, m_{\mu}=105.66 \mathrm{MeV}, m_{\tau}=1.777 \mathrm{GeV}$, $m_{Z}=91.188 \mathrm{GeV}, m_{W}=80.41 \mathrm{GeV}, m_{u}=5 \mathrm{MeV}, m_{c}=1.35 \mathrm{GeV}, m_{t}=174.3 \mathrm{GeV}$, $m_{d}=9 \mathrm{MeV}, m_{s}=150 \mathrm{MeV}, m_{b}=4.3 \mathrm{GeV}, \alpha_{E W}=1 / 128$ 24]. Because our numerical results show that the $R$ corrections are almost independent on $\tan \beta$, we take $\tan \beta=4$ as a representative selection in the $R$ case. The scalar fermion mass terms in lagrangian are written as

$$
-\mathcal{L}_{M_{\tilde{f}}}=\left(\tilde{f}_{L}^{*}, \tilde{f}_{R}^{*}\right) \mathcal{M}_{\tilde{f}}^{2}\left(\begin{array}{c}
\tilde{f}_{L} \\
\tilde{f}_{R}
\end{array}\right)=\left(\tilde{f}_{L}^{*}, \tilde{f}_{R}^{*}\right)\left(\begin{array}{cc}
\mathcal{M}_{\tilde{f} L L}^{2} & \mathcal{M}_{\tilde{f} L R}^{2} \\
\mathcal{M}_{\tilde{f} L R}^{2 *} & \mathcal{M}_{\tilde{f} R R}^{2}
\end{array}\right)\left(\begin{array}{c}
\tilde{f}_{L} \\
\tilde{f}_{R}
\end{array}\right),
$$

The matrix of the scalar fermion mass is

$$
\mathcal{M}_{\tilde{f}}^{2}=\left(\begin{array}{cc}
M_{\tilde{F}_{L}}^{2}+m_{f}^{2}+\cos 2 \beta\left(I_{3}^{f L}-Q_{f} s_{W}^{2}\right) M_{Z}^{2} & m_{f}\left(A_{f}-\mu \kappa_{f}\right) \\
m_{f}\left(A_{f}-\mu \kappa_{f}\right)^{*} & M_{\tilde{F}^{\prime}}^{2}+m_{f}^{2}+\cos 2 \beta Q_{f} s_{W}^{2} M_{Z}^{2}
\end{array}\right),
$$




$$
\left(\kappa_{f}, m_{\tilde{F}_{L}}^{2}, m_{\tilde{F}^{\prime}}^{2}\right)=\left\{\begin{array}{cl}
\left(\cot \beta, m_{\tilde{Q}}^{2}, m_{\tilde{U}}^{2}\right), & \text { when } \tilde{f} \text { is up-squark } \\
\left(\tan \beta, m_{\tilde{Q}}^{2}, m_{\tilde{D}}^{2}\right), & \text { when } \tilde{f} \text { is down-squark } \\
\left(\tan \beta, m_{\tilde{E}_{L}}^{2}, m_{\tilde{E}_{R}}^{2}\right), & \text { when } \tilde{f} \text { is slepton }
\end{array}\right.
$$

where $Q_{f}$ is the charge of scalar fermion, $I_{3}^{f L}$ is the third component of the left-hand fermion weak isospin, $m_{\tilde{Q}}, m_{\tilde{U}}, m_{\tilde{D}}, m_{\tilde{E}_{L}}, m_{\tilde{E}_{R}}$ are the soft-SUSY-breaking masses and $A_{f}$ is the softSUSY-breaking trilinear coupling parameter. If we do not consider the $\mathrm{CP}$ violation, the matrix elements are real and can be diagonalized as

$$
R^{\tilde{f}} \mathcal{M}_{\tilde{f}}^{2} R^{\tilde{f} \dagger}=\operatorname{diag}\left\{m_{\tilde{f}_{1}}^{2}, m_{\tilde{f}_{2}}^{2}\right\}
$$

The mass eigenstates of scalar fermions can be obtained from the transformation of the current eigenstates,

$$
\begin{aligned}
\left(\begin{array}{c}
\tilde{f}_{1} \\
\tilde{f}_{2}
\end{array}\right) & =R^{\tilde{f}}\left(\begin{array}{c}
\tilde{f}_{L} \\
\tilde{f}_{R}
\end{array}\right)=\left(\begin{array}{cc}
\cos \theta_{\tilde{f}} & \sin \theta_{\tilde{f}} \\
-\sin \theta_{\tilde{f}} & \cos \theta_{\tilde{f}}
\end{array}\right)\left(\begin{array}{c}
\tilde{f}_{L} \\
\tilde{f}_{R}
\end{array}\right), \\
\tan 2 \theta_{\tilde{f}} & =\frac{2 \mathcal{M}_{\tilde{f} L R}^{2}}{\mathcal{M}_{\tilde{f} L L}^{2}-\mathcal{M}_{\tilde{f} R R}^{2}}, \\
m_{\tilde{f}_{1,2}}^{2} & =\frac{1}{2}\left\{\mathcal{M}_{\tilde{f} L L}^{2}+\mathcal{M}_{\tilde{f} R R}^{2} \mp \sqrt{\left(\mathcal{M}_{\tilde{f} L L}^{2}-\mathcal{M}_{\tilde{f} R R}^{2}\right)^{2}+4\left(\mathcal{M}_{\tilde{f} L R}^{2}\right)^{2}}\right\} .
\end{aligned}
$$

If we take $\theta_{\tilde{f}}$ as an input parameter, then we get

$$
m_{\tilde{f}_{1,2}}=\sqrt{\frac{1}{2}\left\{\mathcal{M}_{\tilde{f} L L}^{2}+\mathcal{M}_{\tilde{f} R R}^{2} \mp\left|\mathcal{M}_{\tilde{f} L L}^{2}-\mathcal{M}_{\tilde{f} R R}^{2}\right| / \cos 2 \theta_{\tilde{f}}\right\}} .
$$

In our following numerical calculation, we set $\mu=200 \mathrm{GeV}$ and $\tan \beta=4$. In the squark sector, we follow the way in choosing input parameters presented in Ref. [17] and assume $m_{\tilde{Q}^{1,2}}=$ $m_{\tilde{U}^{1,2}}=m_{\tilde{D}^{1,2}}=M_{Q}$ for the first and second generations, $m_{\tilde{Q}^{3}}=m_{\tilde{U}^{3}}=m_{\tilde{D}^{3}}=M_{Q^{3}}$ for the 
third generation, and $\theta_{\tilde{t}}=44.325^{\circ}, \theta_{\tilde{u}, \tilde{d}, \tilde{c}, \tilde{s}, \tilde{b}}=0$. In the slepton sector, we assume $m_{\tilde{E}_{L}^{\alpha}}=$ $m_{\tilde{E}_{R}^{\alpha}}=A_{e^{\alpha}}=M_{L}$, where $e^{1}, e^{2}, e^{3}$ are e, $\mu, \tau$, respectively.

In the numerical calculation for the process $e^{+} e^{-} \rightarrow t \bar{t}$, we take the input data of squark sector describing above and $\left\{M_{Q}, M_{Q^{3}}\right\}=\{300,200\} \mathrm{GeV},\{500,300\} \mathrm{GeV},\{800,500\} \mathrm{GeV}$, respectively. In the calculation of the process $e^{+} e^{-} \rightarrow \gamma \gamma \rightarrow t \bar{t}$, besides the input parameters of squark sector mentioned before, we take $M_{Q^{3}}=200 \mathrm{GeV}$ and $\left\{M_{Q}, M_{L}\right\}=\{300,150\} \mathrm{GeV}$, $\{500,150\} \mathrm{GeV},\{300,600\} \mathrm{GeV},\{500,600\} \mathrm{GeV}$ respectively or otherwise stated. Then the masses of physical squarks and sleptons are obtained from Eqs.(4.1)-(4.8). According to the experimental upper limits of the coupling parameters in the $R$-parity violating interactions presented in Ref. [25], we take the relevant $R$ parameters as $\lambda_{131}^{\prime}=0.05, \lambda_{133}^{\prime}=0.002, \lambda_{233}^{\prime}=0.2$, $\lambda_{132}^{\prime}=\lambda_{231}^{\prime}=\lambda_{232}^{\prime}=\lambda_{331}^{\prime}=\lambda_{332}^{\prime}=\lambda_{333}^{\prime}=0.4$ for numerical representation.

The cross section of the process $e^{+} e^{-} \rightarrow t \bar{t}$ as a function of $\sqrt{\hat{s}}$ is plotted in Fig.3, where $R$-parity conserving (RC) and R-parity violating (RV) results are presented. The four curves correspond to (1) R-parity conserving case, (2) R-parity violating case with $M_{Q}=300 \mathrm{GeV}$ and $M_{Q^{3}}=200 \mathrm{GeV}$, (3) R-parity violating case with $M_{Q}=500 \mathrm{GeV}$ and $M_{Q^{3}}=300 \mathrm{GeV}$, (4) R-parity violating case with $M_{Q}=800 \mathrm{GeV}$ and $M_{Q^{3}}=500 \mathrm{GeV}$, respectively. We can see that the $R$ effect on the production cross section decreases with the increments of $M_{Q}$ and $M_{Q^{3}}$, and if the masses of squarks are about $200 \mathrm{GeV}$ and $300 \mathrm{GeV}$ and $\sqrt{\hat{s}}=500 \mathrm{GeV}$, the relative $R$ correction $\left(\delta=\frac{\Delta \sigma}{\sigma_{0}}\right)$ can reach about $-30 \%$. So we can say that if $R$ really exists, the $R$ effect on the cross section of the process $e^{+} e^{-} \rightarrow t \bar{t}$ can be observed or its accurate measurement can provide more stringent constraints on the masses of squarks, sleptons and $\lambda^{\prime}$. 
Fig.4-8 demonstrate the $R$ and $R$-conserving SUSY EW-like one-loop corrections to $e^{+} e^{-} \rightarrow$ $\gamma \gamma \rightarrow t \bar{t}$. Fig.4(a) presents the dependence of the $R$ and $R$-conserving SUSY EW-like one-loop corrections $\Delta \sigma$ on the $\sqrt{\hat{s}}$ for the subprocess $\gamma \gamma \rightarrow t \bar{t}$. Fig.4(b) presents the dependence of the $R$ and $R$-conserving SUSY EW-like one-loop relative corrections $\delta=\Delta \sigma / \sigma_{\text {tree }}$ on the $\sqrt{\hat{s}}$. In Fig.4(a-b), we have $m_{\tilde{Q}^{3}}=200 \mathrm{GeV}$. The solid line is for $M_{Q}=300 \mathrm{GeV}, M_{L}=150 \mathrm{GeV}$, the dashed line is for $M_{Q}=500 \mathrm{GeV}, M_{L}=150 \mathrm{GeV}$. The dotted and dash-dotted lines are for $M_{Q}=300 \mathrm{GeV}, M_{L}=600 \mathrm{GeV}$ and $M_{Q}=500 \mathrm{GeV}, M_{L}=600 \mathrm{GeV}$, respectively, and dash-dot-dotted line for the corresponding $R$-conserving SUSY EW-like corrections. On all the curves in both Fig.4(a) and Fig.4(b), we can see line structures with small spikes due to the resonance effects. For example, on some of the curves in Fig.4(a) there exist small resonance spikes in the region around the vicinities of $\sqrt{\hat{s}} \sim 2 m_{\tilde{b}_{2}} \sim 415 \mathrm{GeV}$ and $\sqrt{\hat{s}} \sim 2 m_{\tilde{b}_{1}} \sim 403 \mathrm{GeV}$. On the curves of $M_{Q}=300 \mathrm{GeV}, M_{L}=150 \mathrm{GeV}$ and $M_{Q}=300 \mathrm{GeV}, M_{L}=600 \mathrm{GeV}$, we can see other resonance effect at $\sqrt{\hat{s}} \sim 2 m_{\tilde{d}_{1}, \tilde{s}_{1}} \sim 602 \mathrm{GeV}$ and $\sqrt{\hat{s}} \sim 2 m_{\tilde{d}_{2}, \tilde{s}_{2}} \sim 610 \mathrm{GeV}$. For the curves of $M_{Q}=300 \mathrm{GeV}, M_{L}=600 \mathrm{GeV}$ and $M_{Q}=500 \mathrm{GeV}, M_{L}=600 \mathrm{GeV}$, the resonance effect can be seen around the position of $\sqrt{\hat{s}} \sim 2 m_{\tilde{e}_{1,2}, \tilde{\mu}_{1,2}, \tilde{\tau}_{1,2}} \sim 1203 \mathrm{GeV}$. The corresponding line structures due to resonance effect are shown again in Fig.4(b). The $R$ effects shown in these two figures are very obvious, all the curves corresponding to the different input $M_{L}$ and $M_{Q}$ values demonstrate that the $R$ corrections are comparable to the $R$-conserving SUSY oneloop EW-like corrections. Especially for the curves with $\left\{M_{Q}=300 \mathrm{GeV}, M_{L}=150 \mathrm{GeV}\right\}$ and $\left\{M_{Q}=500 \mathrm{GeV}, M_{L}=150 \mathrm{GeV}\right\}$, the $R$ corrections are larger than the corresponding one-loop $R$-conserving SUSY one and the $R$ relative correction for $\left\{M_{Q}=300 \mathrm{GeV}, M_{L}=150 \mathrm{GeV}\right\}$ 
can reach $-4.1 \%$.

In Fig.5 and Fig. 6 we take $M_{Q^{3}}=200 \mathrm{GeV}, \sqrt{\hat{s}}=500 \mathrm{GeV}$ and depict the $R$ relative corrections as the functions of $M_{Q}$ and $M_{L}$ respectively. In Fig.5 the curves correspond to $M_{L}=150 \mathrm{GeV}, 300 \mathrm{GeV}, 500 \mathrm{GeV}$ and $800 \mathrm{GeV}$, respectively, and $M_{Q}$ varies from $300 \mathrm{GeV}$ to $900 \mathrm{GeV}$. We can see from Fig.5 that the corrections are sensitive to the value of $M_{Q}$ when squark mass parameter $M_{Q}$ is below $400 \mathrm{GeV}$, typically when $M_{Q}=300 \mathrm{GeV}$ the relative correction can reach $-3.0 \%$. While when $M_{Q}$ is larger than $400 \mathrm{GeV}$, the relative corrections are not sensitive to $M_{Q}$ due to the decouple theorem. In Fig.6 we choose $M_{Q}=300 \mathrm{GeV}, 500 \mathrm{GeV}$ and $800 \mathrm{GeV}$ respectively, $M_{L}$ goes from $100 \mathrm{GeV}$ to $900 \mathrm{GeV}$. The spikes at $M_{L} \sim 245 \mathrm{GeV}$ are from the resonance effect because the relation of $\sqrt{\hat{s}} \sim 2 m_{\tilde{e}_{1,2}, \tilde{\mu}_{1,2}, \tilde{\tau}_{1,2}} \sim 500 \mathrm{GeV}$ exists. If $M_{L}$ is below $200 \mathrm{GeV}$, for all taken $M_{Q}$ values in the figure, the corrections are relative large and can reach $-3.0 \%$. But when $M_{L}>300 \mathrm{GeV}$, the $R$ corrections are not sensitive to $M_{L}$.

In Fig.7 we have $\sqrt{\hat{s}}=500 \mathrm{GeV}, M_{Q^{3}}=200 \mathrm{GeV}$ and take (1) $M_{Q}=300 \mathrm{GeV}, M_{L}=$ $150 \mathrm{GeV}$ (solid line); (2) $M_{Q}=500 \mathrm{GeV}, M_{L}=150 \mathrm{GeV}$ (dashed line); (3) $M_{Q}=300 \mathrm{GeV}, M_{L}=$ $600 \mathrm{GeV}$ (dotted line) and $(4) M_{Q}=500 \mathrm{GeV}, M_{L}=600 \mathrm{GeV}$ (dash-dotted line), respectively, assuming $\lambda_{132}^{\prime}=\lambda_{231}^{\prime}=\lambda_{232}^{\prime}=\lambda_{331}^{\prime}=\lambda_{332}^{\prime}=\lambda_{333}^{\prime}=\lambda^{\prime}$. The relative $R$ corrections to the subprocess $\gamma \gamma \rightarrow t \bar{t}$ as the functions of $\lambda^{\prime}$ are plotted in this figure. We can see that the $R$ corrections are negative and reduce the cross section of the subprocess. The figure shows that the relative corrections are getting larger with the increment of the $\lambda^{\prime}$ value when sleptons have small masses, but the $R$ effects would be very weak if sleptons are heavy. When we have $\lambda^{\prime}=0.6$ and $M_{Q}=300 \mathrm{GeV}, M_{L}=150 \mathrm{GeV}$, the relative correction can reach $-6.6 \%$. 
Fig.8(a) shows the one-loop $R$ corrections to the parent process $e^{+} e^{-} \rightarrow \gamma \gamma \rightarrow t \bar{t}$ as the functions of the electron-positron colliding energy. We take again the input parameter sets as: (1) $M_{Q}=300 \mathrm{GeV}, M_{L}=150 \mathrm{GeV}$ (full line); (2) $M_{Q}=500 \mathrm{GeV}, M_{L}=150 \mathrm{GeV}$ (dashed line); (3) $M_{Q}=300 \mathrm{GeV}, M_{L}=600 \mathrm{GeV}\left(\right.$ dotted line) and (4) $M_{Q}=500 \mathrm{GeV}, M_{L}=600 \mathrm{GeV}($ dashdotted line), respectively, and vary the $e^{+} e^{-}$colliding energy from 0 to $2 \mathrm{TeV}$. At the position of $\sqrt{s} \sim 1.2 \mathrm{TeV}$ the absolute corrections reach their maximal values, e.g., for the curve of $M_{Q}=300 \mathrm{GeV}, M_{L}=150 \mathrm{GeV}$, the curve has the maximal correction $\Delta \sigma_{\max }=-15.5 \mathrm{fb}$. When $\sqrt{s}>1.2 \mathrm{TeV}$, the absolute corrections decrease with the increment of the colliding c.m.s energy. Fig.8(b) shows the one-loop $R$ and $R$-conserving SUSY electroweak relative corrections with $M_{Q^{3}}=200 \mathrm{GeV}$ as the functions of the colliding $e^{+} e^{-}$energy. We can see that when the colliding energy is below $1.2 \mathrm{TeV}$, the $R$ absolute relative corrections decrease apparently with the increment of $\sqrt{s}$ except the curve with heavy masses of sleptons and the first and second generation squarks $\left(M_{Q}=500 \mathrm{GeV}, M_{L}=600 \mathrm{GeV}\right)$. But when $\sqrt{s}$ is larger than $1.2 \mathrm{TeV}$, the $R$ relative corrections are not very sensitive to the c.m.s energy $\sqrt{s}$. In comparison with the $R$-conserving one-loop SUSY electroweak corrections, we can conclude that the $R$ relative corrections are comparable or even larger than the $R$-conserving one-loop SUSY electroweak relative corrections for almost all the input parameters we used in these two figures. When $M_{Q}=300 \mathrm{GeV}, M_{L}=150 \mathrm{GeV}$, the relative correction can reach $-3.6 \%$. 


\section{Summary}

In this paper, we studied the effect of the $R$-parity lepton number violation in the MSSM on both important processes $e^{+} e^{-} \rightarrow t \bar{t}$ and $e^{+} e^{-} \rightarrow \gamma \gamma \rightarrow t \bar{t}$ at a LC. To the former process, we find that the $R$ effect is obviously related to the masses of squark and slepton. The heavier the squarks and sleptons are, the smaller the $R$ effect is. The $R$ relative correction can reach $-30 \%$ with the favorable parameters. To the second process, our calculation shows that the $R$ corrections to either subprocess $\gamma \gamma \rightarrow t \bar{t}$ or parent process $e^{+} e^{-} \rightarrow \gamma \gamma \rightarrow t \bar{t}$ are strongly related to the colliding energy. The $R$ relative correction can reach several percent to both cross sections of the subprocess and parent process. Although the $R$ correction is smaller than QCD correction [14, it can be even larger than the $R$-conserving SUSY electroweak correction with suitable parameters [15] [16] [17. So the $R$ effect on both processes could be significant and could be measured experimentally, if the $R$ really exists. We also investigate the dependence of the $R$

correction on the relevant $R$ input parameters, such as $M_{L}, M_{Q}, M_{Q^{3}}, \lambda_{i j k}^{\prime}$, etc. We find that the $R$ correction is strongly related to the input parameters $M_{Q}, M_{Q^{3}}, M_{L}$ and $\lambda_{i j k}^{\prime}$ in some parameter space, but is not sensitive to squark mass (or slepton mass) when $m_{\tilde{q}} \geq 400 \mathrm{GeV}$ (or $m_{\tilde{l}} \geq 300 \mathrm{GeV}$ ) and is almost independent on $\tan \beta$.

Acknowledgments: This work was supported in part by the National Natural Science Foundation of China and a grant from the University of Science and Technology of China.

\section{Appendix}

The relevant Feynman rules of R-parity violating interactions are showed as below 


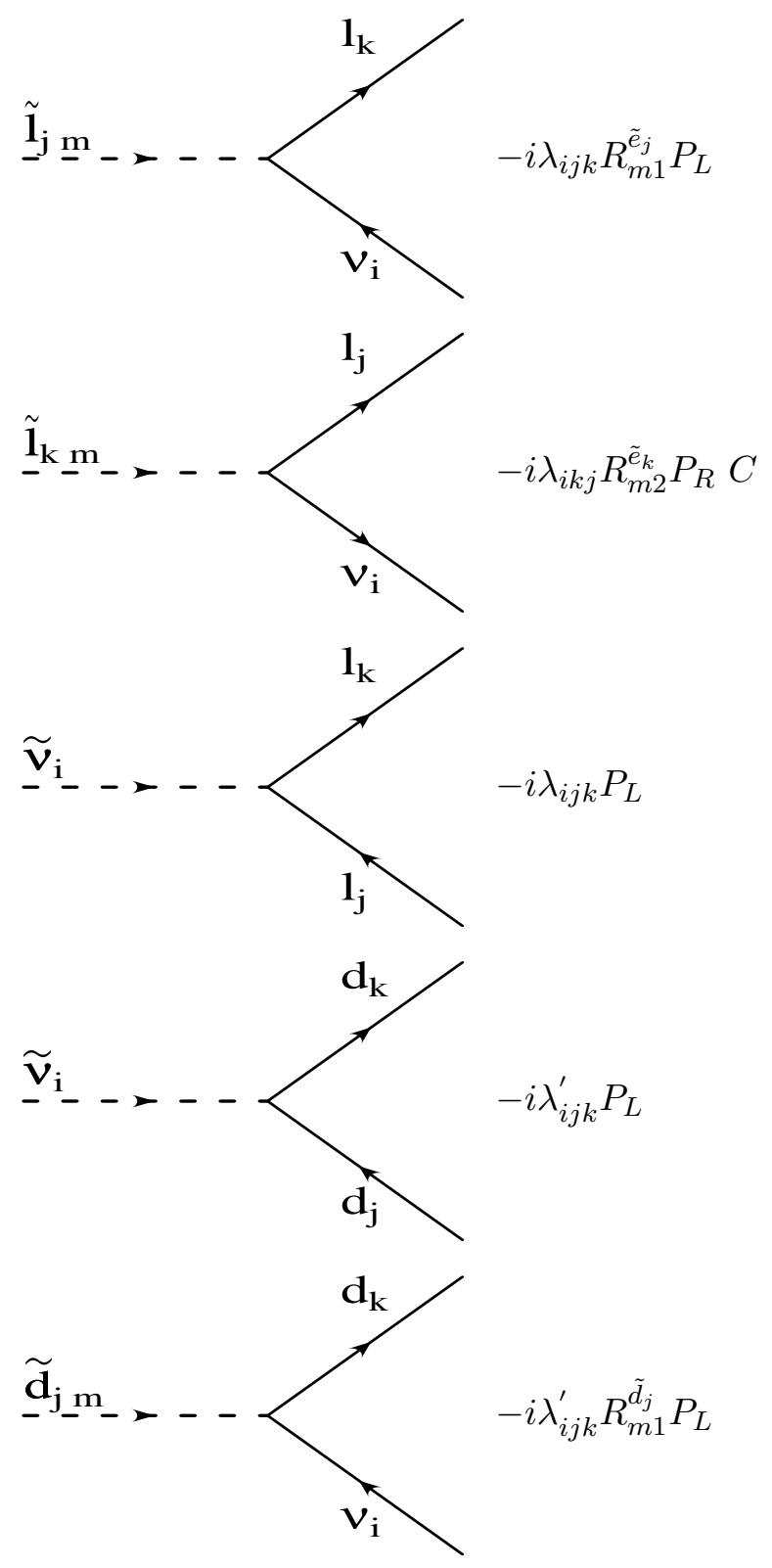



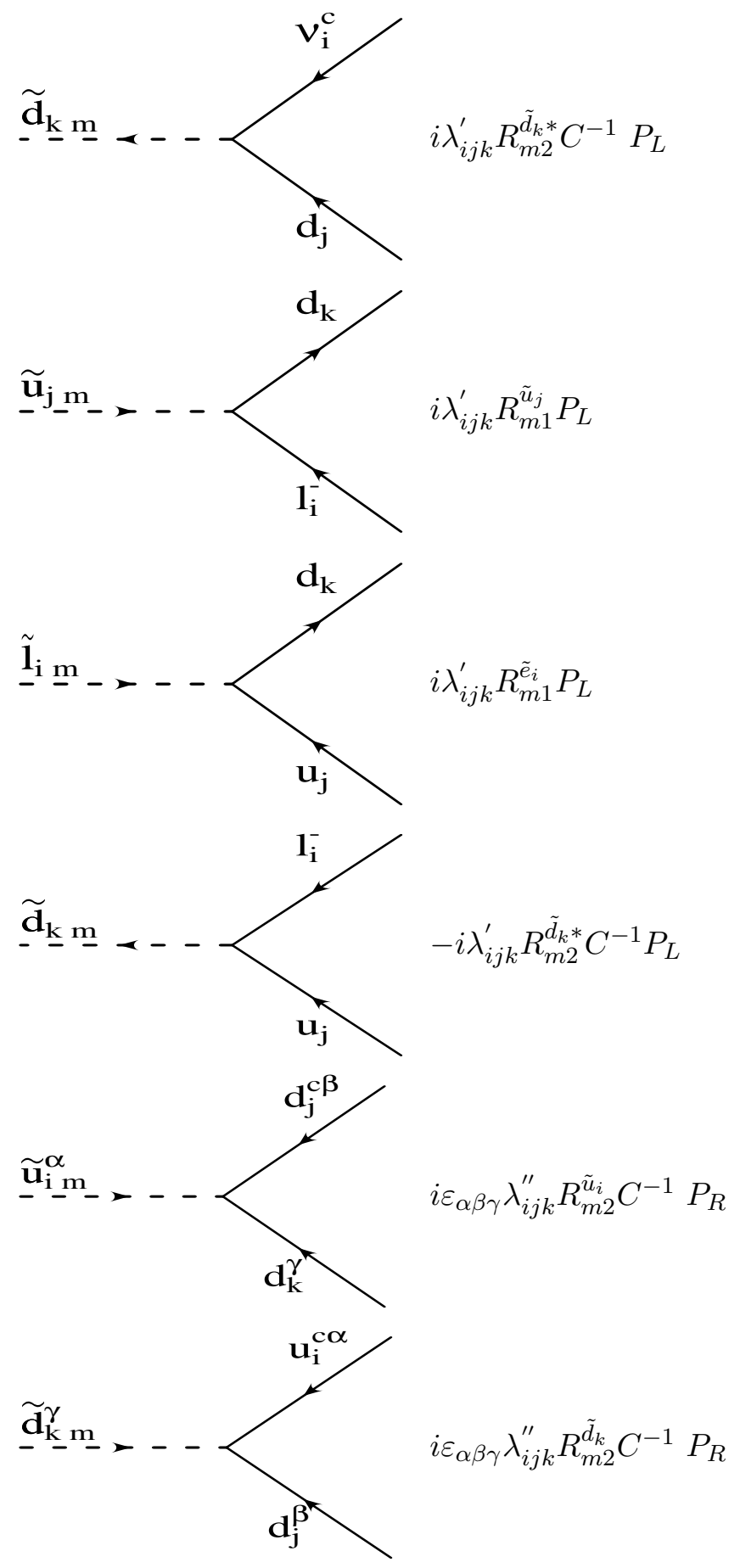


\section{References}

[1] H. E. Haber and G. L. Kane, Phys. Rep. 117 (1985) 75.

[2] Yin Jun, Ma Wen-Gan, Wan Lang-Hui and Zhang Ren-You, Phys. Rev. D65 (2002) 116006

[3] J. L. Goity and M. Sher, Phys. Lett B. 346 (1996) 95.

[4] Y. Totsuka, in Proc. of the XXIV conf. on High Energy Physics, Munich, 1988.

[5] J. L. Goity and M. Sher, Phys. Lett B. 346 (1995) 69.

[6] C. Adolphsen et al. (International Study Group Collaboration), "International study group progress report on linear collider development," SLAC-R-559 and KEK-REPORT-2000-7 (April, 2000).

[7] N. Akasaka et al., "JLC design study," KEK-REPORT-97-1

[8] R. Brinkmann, K. Flottmann, J. Rossbach, P. Schmuser, N. Walker and H. Weise(editor), "TESLA: The superconducting electron positron linear collider with an integrated X-ray laser laboratory. Technical design report, Part 2: The Accelerator," DESY-01-11 (March, 2001).

[9] "A $3 \mathrm{TeV} e^{+} e^{-}$Linear Collider Based on CLIC Technology", G.Guignard(editor), CERN2000-008. 
[10] I.F. Ginzburg, G.L. Kotkin, V.G. Serbo and V.I. Telov, Nucl. Instrum. Meth. Nucl. Instrum. Meth. A205, 47(1983); I.F. Ginzburg, G.L. Kotkin, V.G. Panfil, V.G. Serbo and V.I. Telov, Nucl. Instrum. Meth. A219, 5(1984);

[11] Ma Wen-Gan, C. S. Li, and Han Liang, Phys. Rev. D53, 1304 (1996).

[12] W. Hollik and C. Schappacher, Nucl. Phys. B545, 98 (1999).

[13] Anindya Datta, Phys. Rev. D65(2002) 054019, hep-ph/0108150.

[14] Han Liang, Ma Wen-Gan, and Yu Zeng-Hui, Phys. Rev. D56, 265 (1997)

[15] A. Denner, S. Dittmaier, and M. Strobel, Phys. Rev. D53, 44 (1996)

[16] C. S. Li, J. M. Yang, Y. L. Zhu, and H. Y. Zhou, Phys. Rev. D54, 4662 (1996).

[17] Zhou Mian-Lai, Ma Wen-Gan, Han Liang, Jiang Yi and Zhou Hong, Phys. Rev. D61, (2000) 033008.

[18] P. Roy, TIFR/TH/97-60; D. K. Ghosh, S. Raychaudburi and K. Sridbar, Phys. Lett. B396(1997)177; Yin Xi, Ma Wen-Gan, Wan Lang-Hui, Han Liang and Jiang Yi, Comm. Theor. Phys. 36 (2001) 553.

[19] Bernd A. Kniehl, Phys. Rep. 240(1994)211.

[20] D. M. Copper, D. R. T. Jones, and P. van Nieuwenhuizen, Nucl. Phys. B167, 479 (1980); W. Siegel, Phys. Lett. 84B, 193 (1979)

[21] D. A. Ross and J. C. Taylor, Nucl. Phys. B51, 25 (1979). 
[22] G. Passarino and M. Veltman, Nucl. Phys. B160, 151(1979).

[23] V. Telnov, Nucl. Instrum. Methods Phys. Res. A294(1990)72; L. Ginzburg, G. Kotkin and H. Spiesbergerm, Fortschr. Phys. 34(1986)687.

[24] Particle Data Group, Eur. Phys. J. C15 2000.

[25] B. Allanach, H. Baer, S. Banerjee, E. L. Berger et al., hep-ph/9906224, and the references herein; B. C. Allanach, A. Dedes, and H. K. Dreiner, Phys. Rev. D60,(1999) 075014.

\section{Figure Captions}

Fig.1 The relevant Feynman diagrams for the process $e^{+} e^{-} \rightarrow t \bar{t}$ in the MSSM at the treelevel: (a)the Feynman diagrams for R-parity conserved MSSM part; (b)the Feynman diagrams for R-parity violation MSSM part.

Fig.2 The relevant Feynman diagrams for the subprocess $\gamma \gamma \rightarrow t \bar{t}$ in the MSSM with $R$-parity lepton number violation at the tree-level and the one-loop level diagrams with $R$ interactions: Fig.2(a) is tree level diagram. Fig.2(b.1)-(b.8) are vertex diagrams. Fig.2(c.1)-(c.6) are box diagrams. Fig.2(d.1)-(d.2) and Fig.2(f.1)-(f.2) are self-energy diagrams. Fig.2(e.1)-(e.2) are quartic coupling diagrams.

Fig.3 The cross section of the process $e^{+} e^{-} \rightarrow t \bar{t}$ as a function of $\sqrt{\hat{s}}$.

Fig.4(a) The one-loop $R$ and $R$-conserving SUSY EW-like corrections $\Delta \sigma$ as the functions of $\sqrt{\hat{s}}$ for the subprocess $\gamma \gamma \rightarrow t \bar{t}$. 
Fig.4(b) The one-loop $R$ and $R$-conserving SUSY EW-like relative corrections $\delta=\Delta \sigma / \sigma_{\text {tree }}$ as the functions of $\sqrt{\hat{s}}$ for the subprocess $\gamma \gamma \rightarrow t \bar{t}$.

Fig.5 The one-loop $R$ relative corrections to the subprocess $\gamma \gamma \rightarrow t \bar{t}$ as the functions of $M_{Q}$ with $\sqrt{\hat{s}}=500 \mathrm{GeV}$.

Fig.6 The one-loop $R$ relative corrections to the subprocess $\gamma \gamma \rightarrow t \bar{t}$ as the functions of $M_{L}$ with $\sqrt{\hat{s}}=500 \mathrm{GeV}$.

Fig.7 The one-loop $R$ relative corrections to the subprocess $\gamma \gamma \rightarrow t \bar{t}$ as the functions of $\lambda^{\prime}$ with $\sqrt{\hat{s}}=500 \mathrm{GeV}$ (We assume $\lambda_{132}^{\prime}=\lambda_{231}^{\prime}=\lambda_{232}^{\prime}=\lambda_{331}^{\prime}=\lambda_{332}^{\prime}=\lambda_{333}^{\prime}=\lambda^{\prime}$ ).

Fig.8(a) The one-loop $R$ corrections $\Delta \sigma$ to the parent process $e^{+} e^{-} \rightarrow \gamma \gamma \rightarrow t \bar{t}$ as the functions of the c.m.s energy of the incoming electron-positron pair.

Fig.8(b) The one-loop $R$ and $R$-conserving SUSY EW-like relative corrections to the parent process $e^{+} e^{-} \rightarrow \gamma \gamma \rightarrow t \bar{t}$ as the functions of the c.m.s energy of the incoming electron-positron pair. 


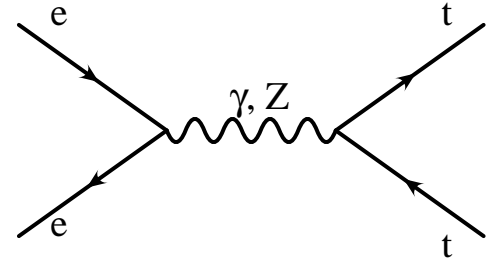

(a)

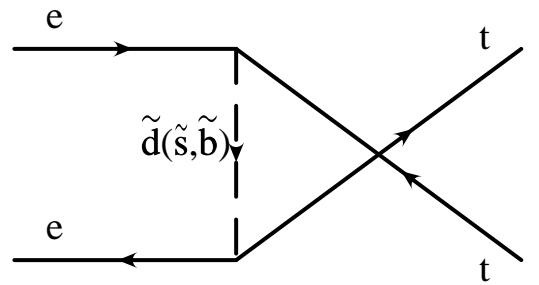

(b)

Fig.1 


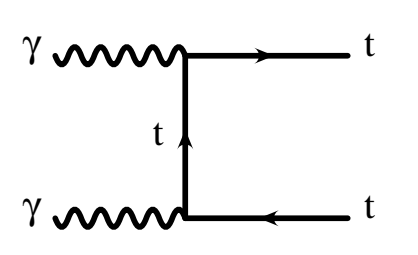

(a)

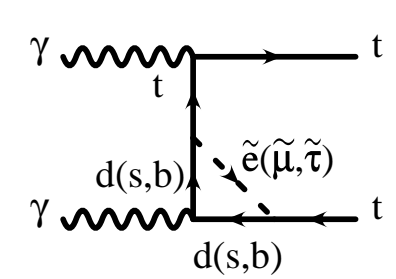

(b.1)

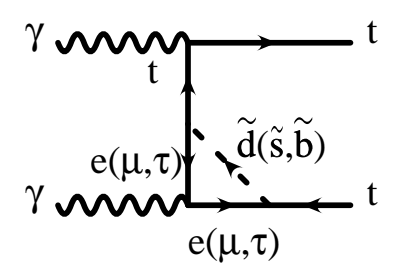

(b.2)

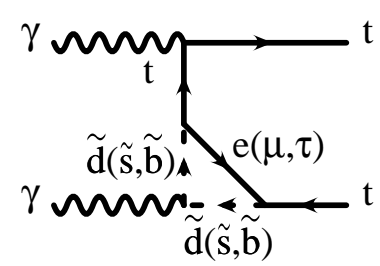

(b.3)

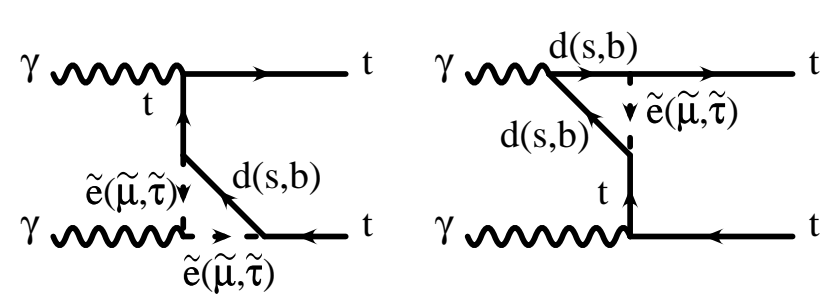

(b.4)

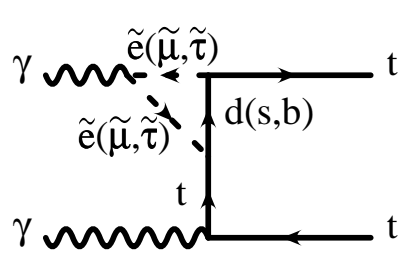

(b.8)

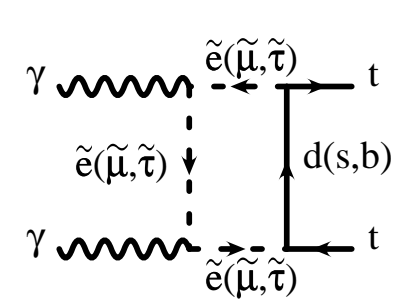

(c.4)

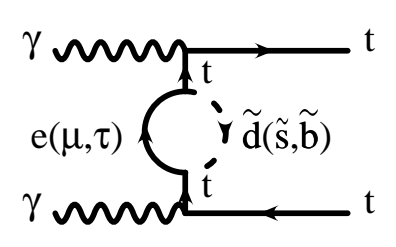

(d.2)
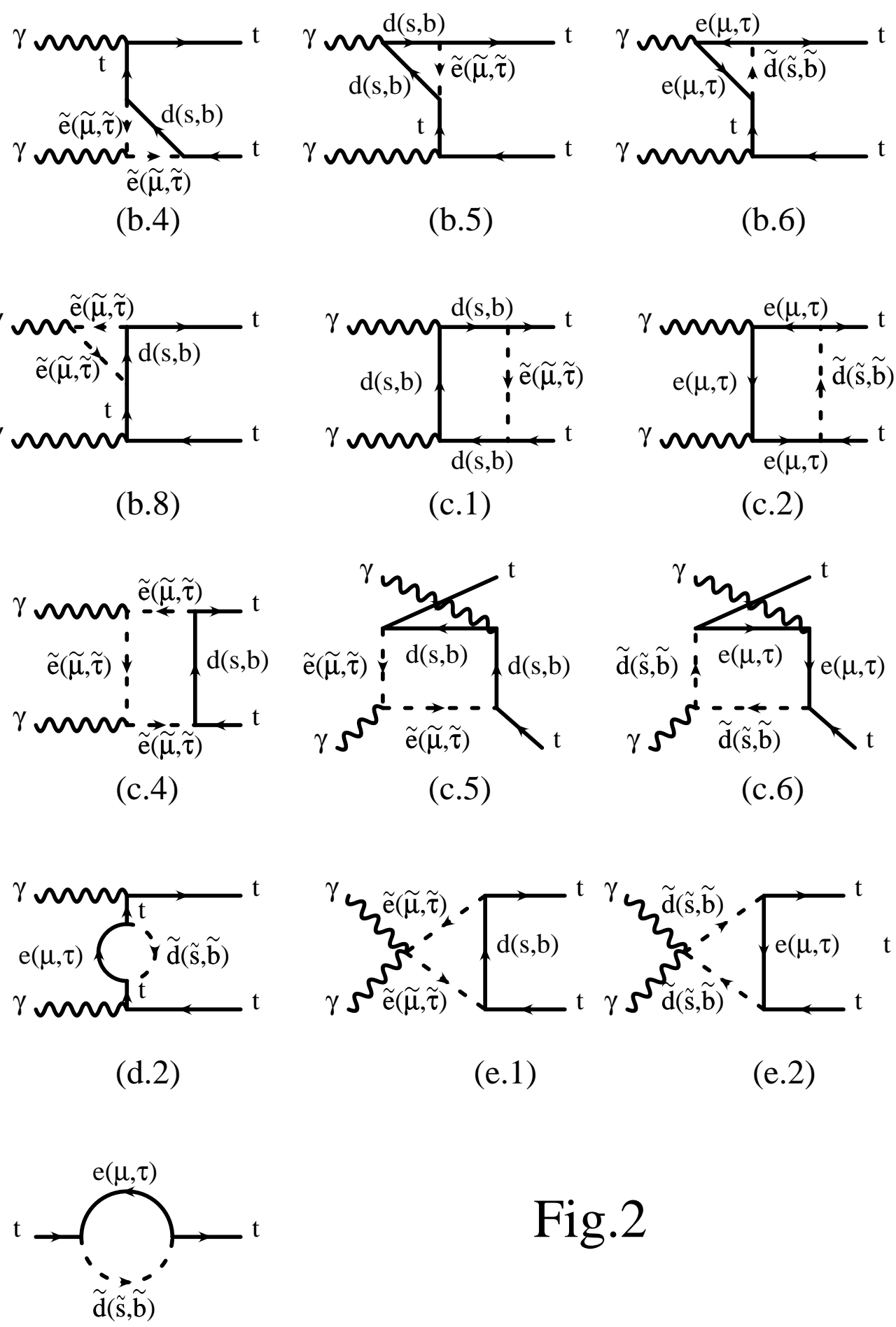

(b.5)

(b.6)

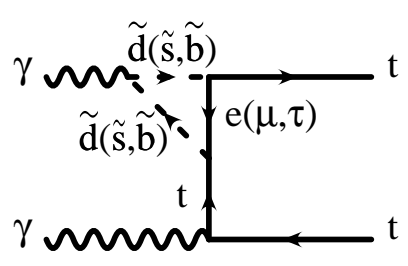

(b.7)

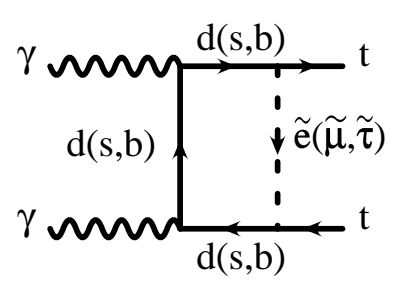

(c.1)

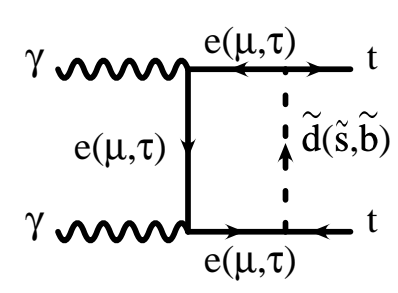

(c.2)

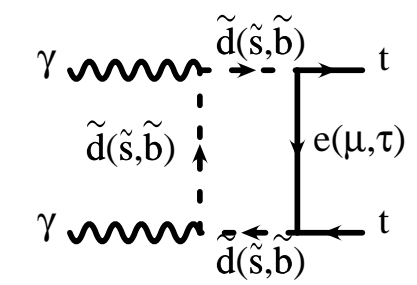

(c.3)

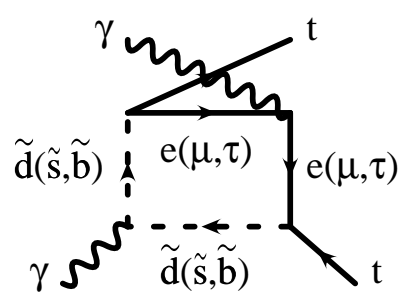

(c.6)

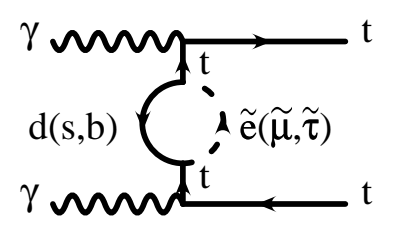

(d.1)
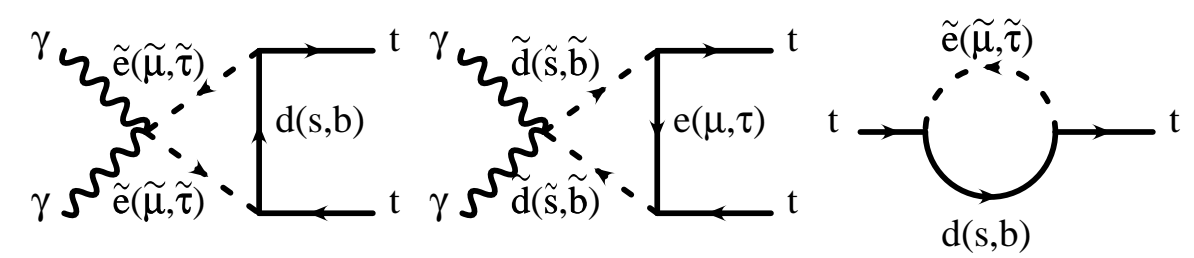

(e.1)

(e.2)

(f.1) 
Fig.3

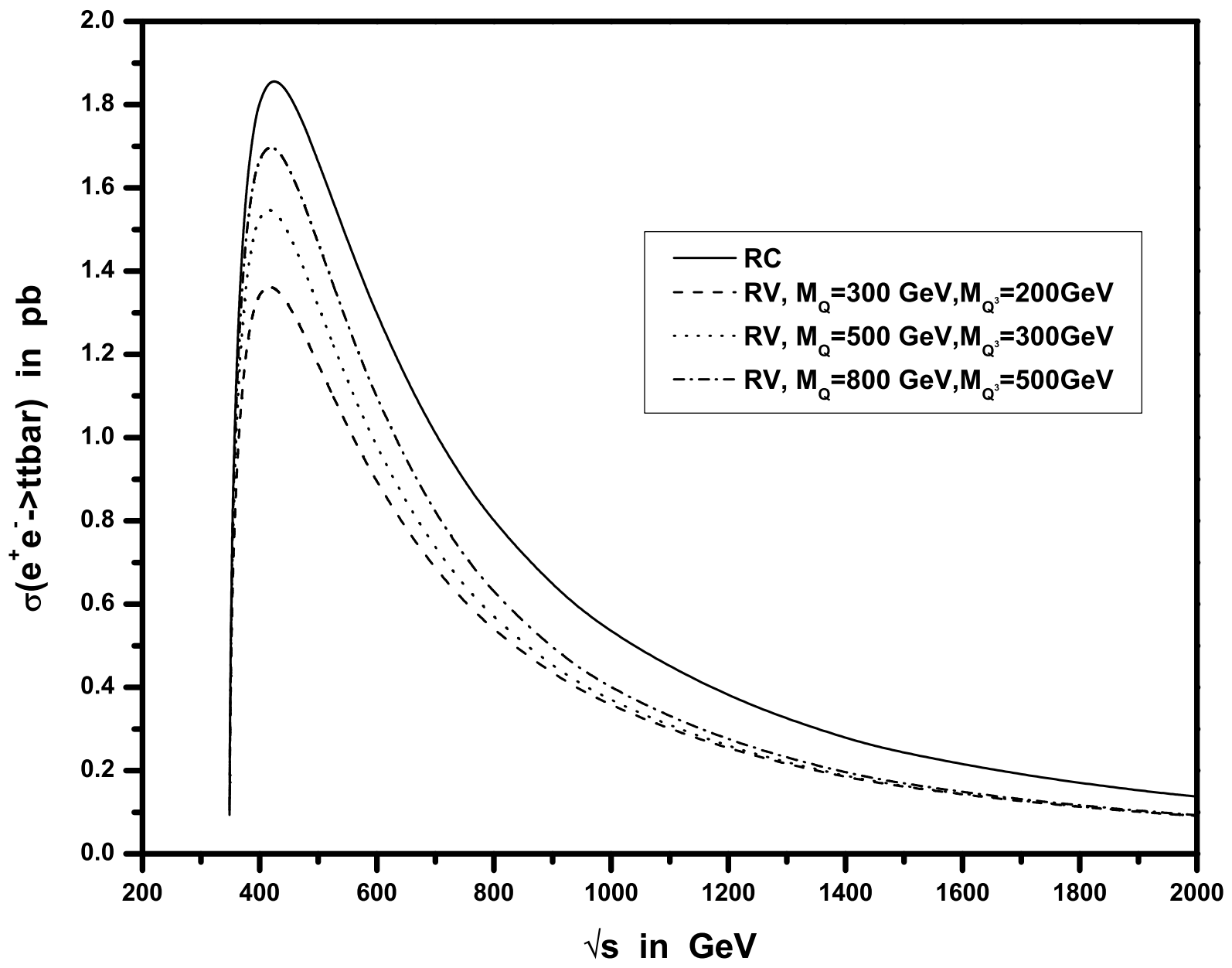


Fig.4(a)

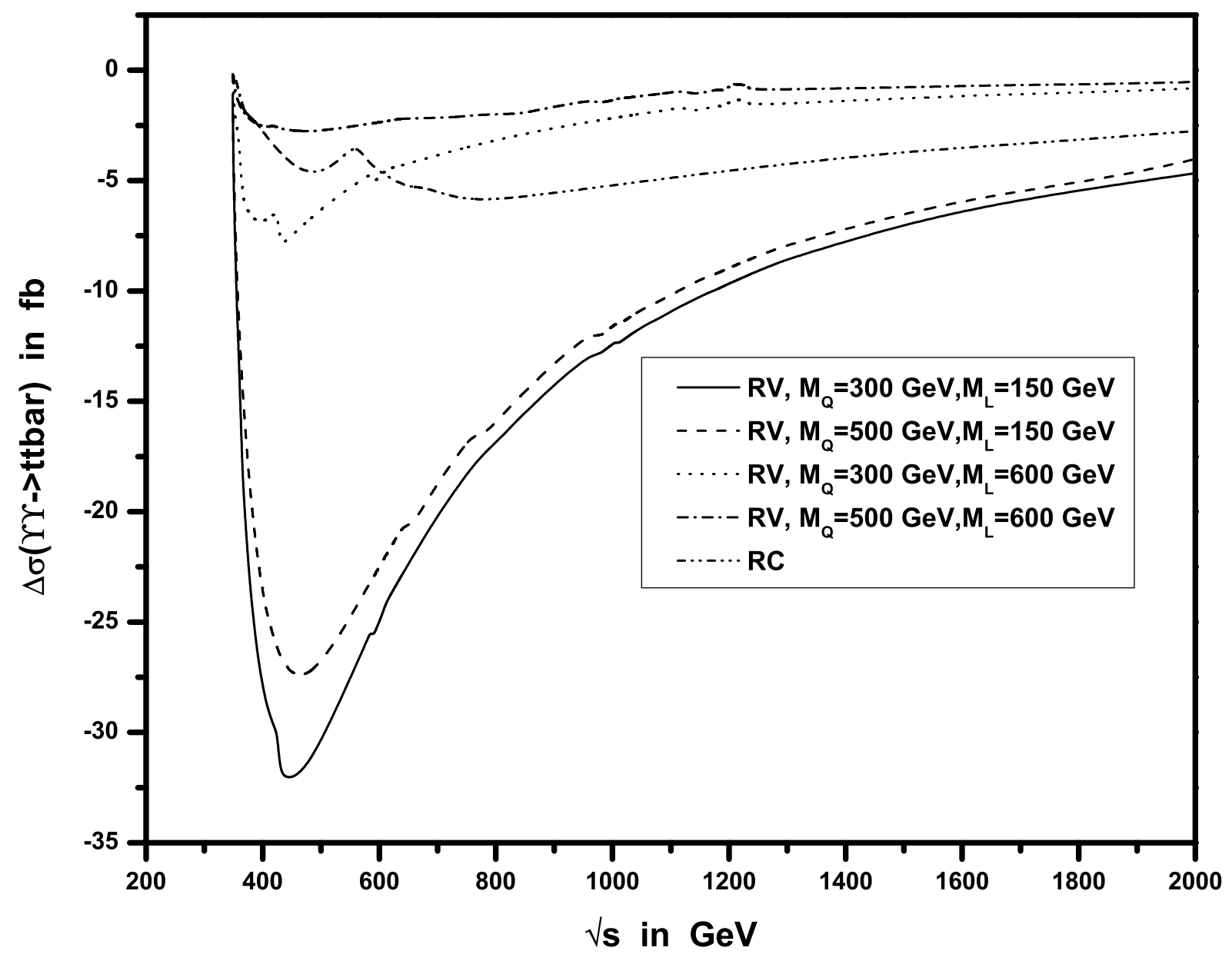


Fig.4(b)

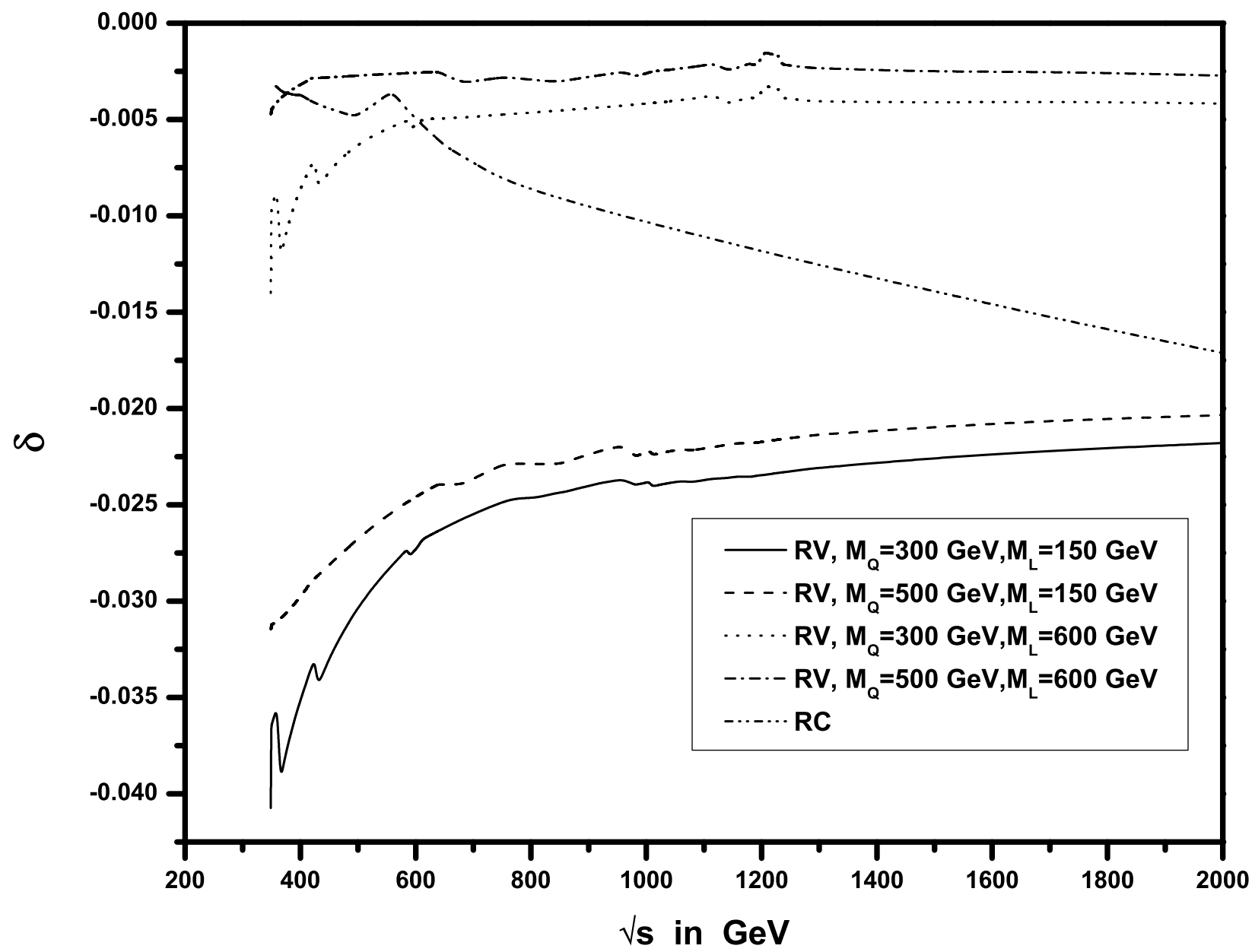


Fig.5

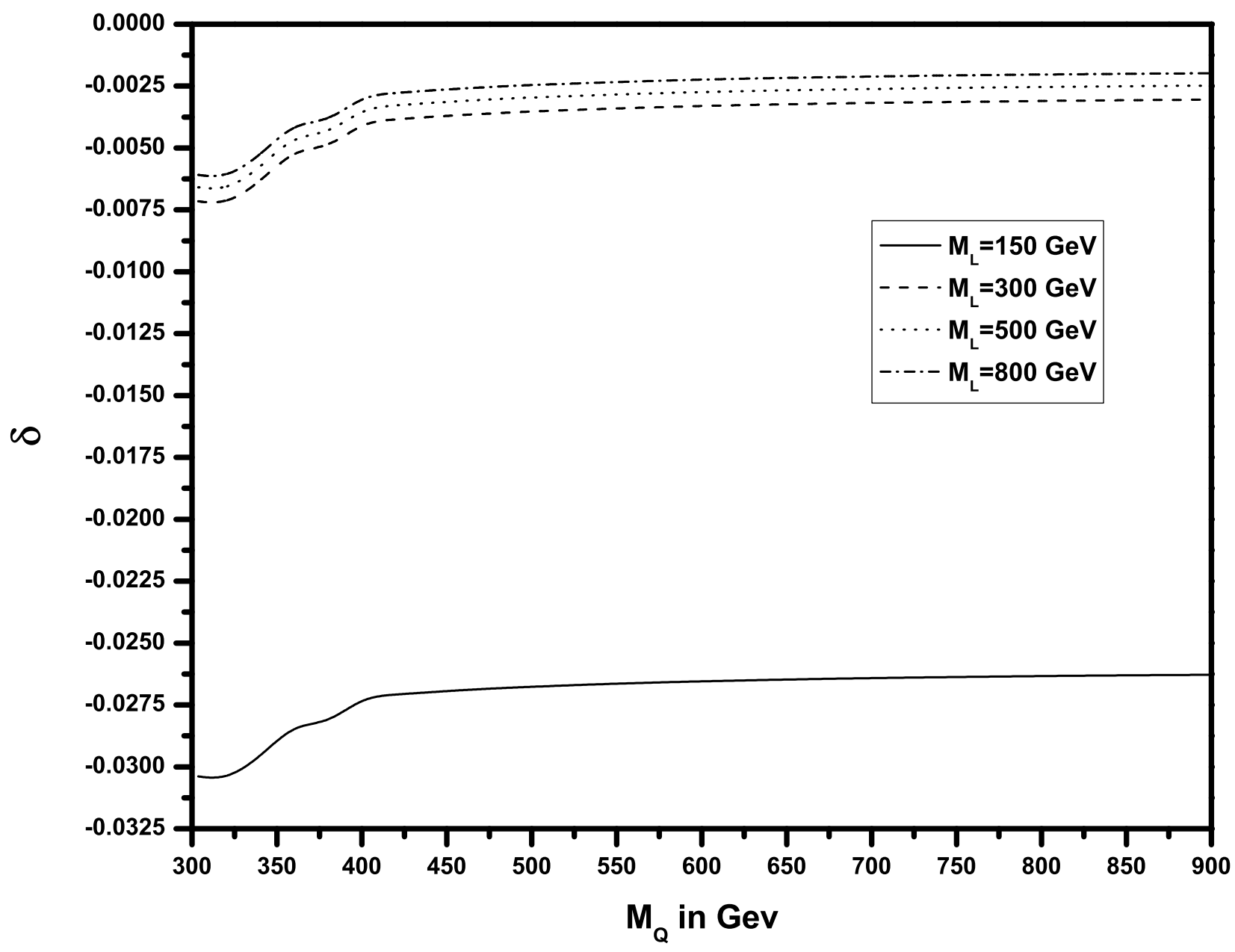


Fig. 6

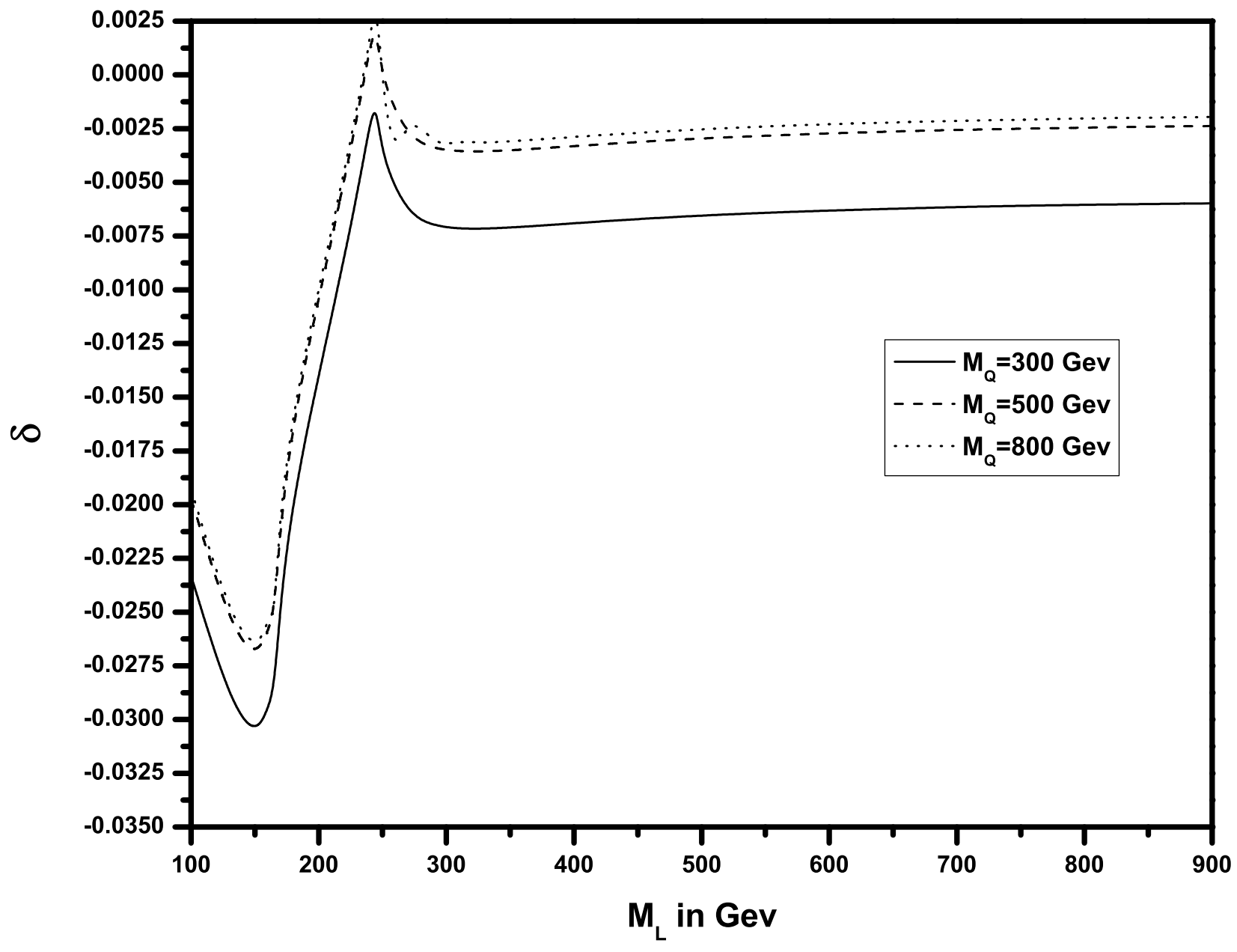


Fig.7

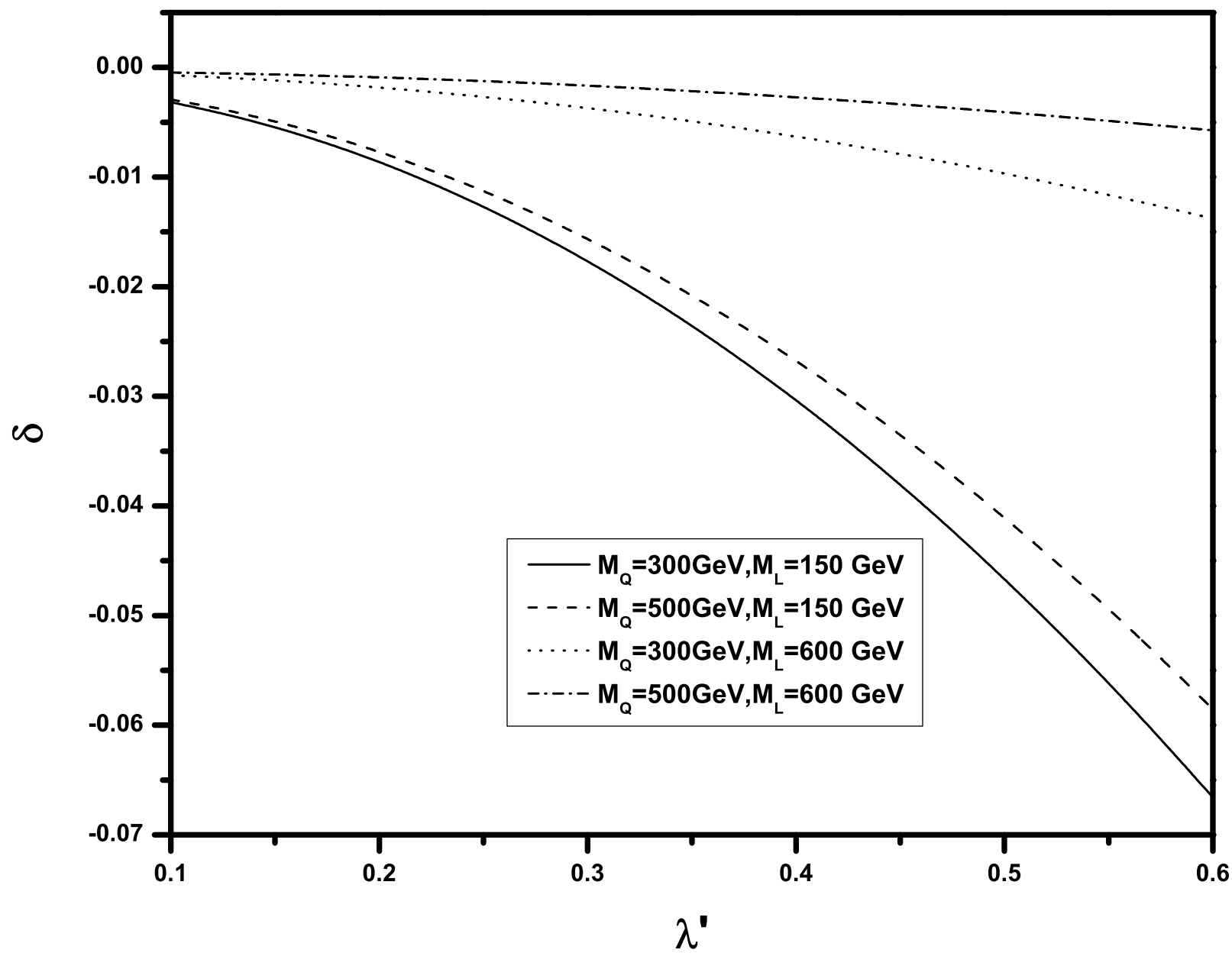


Fig.8(a)

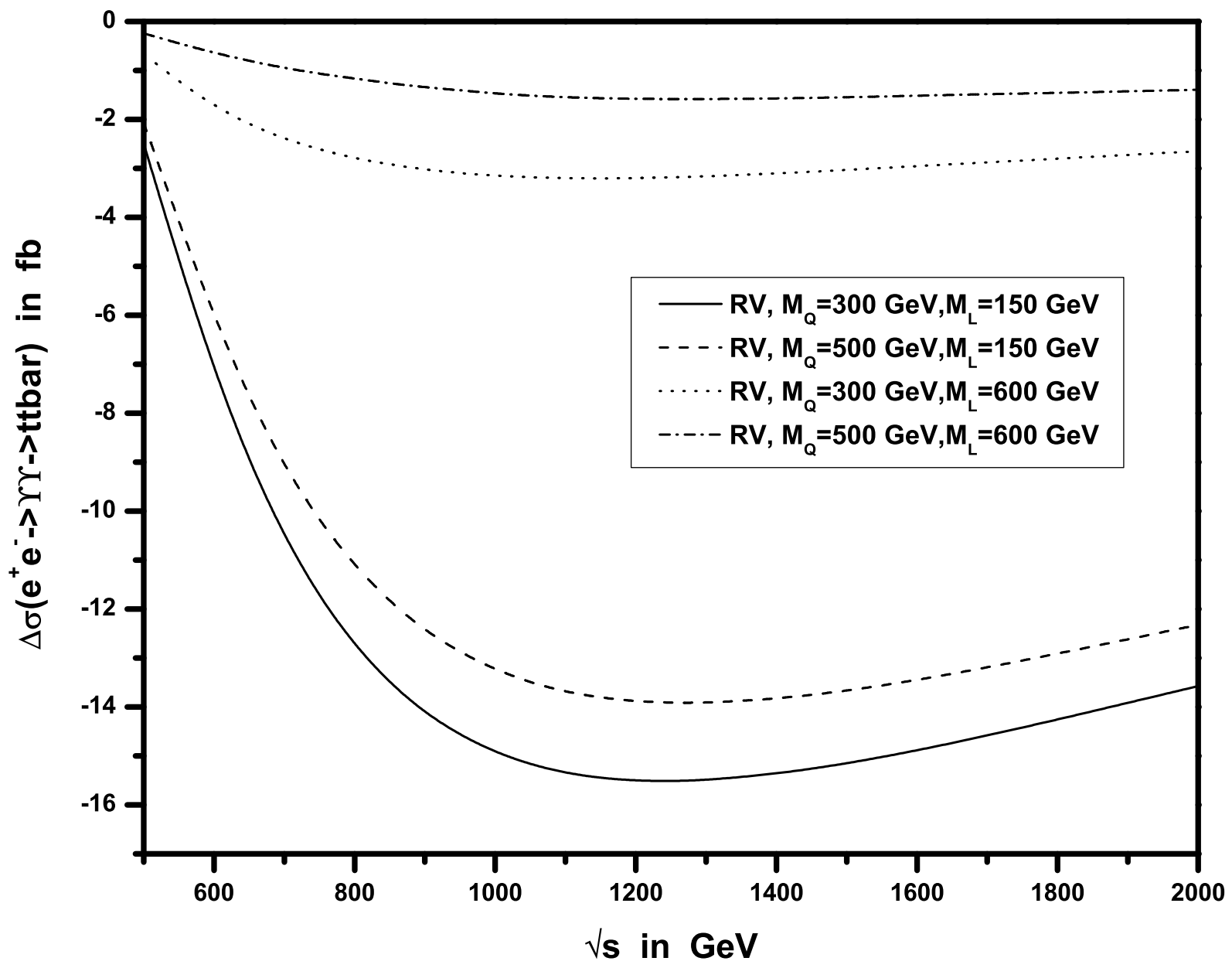


Fig.8(b)

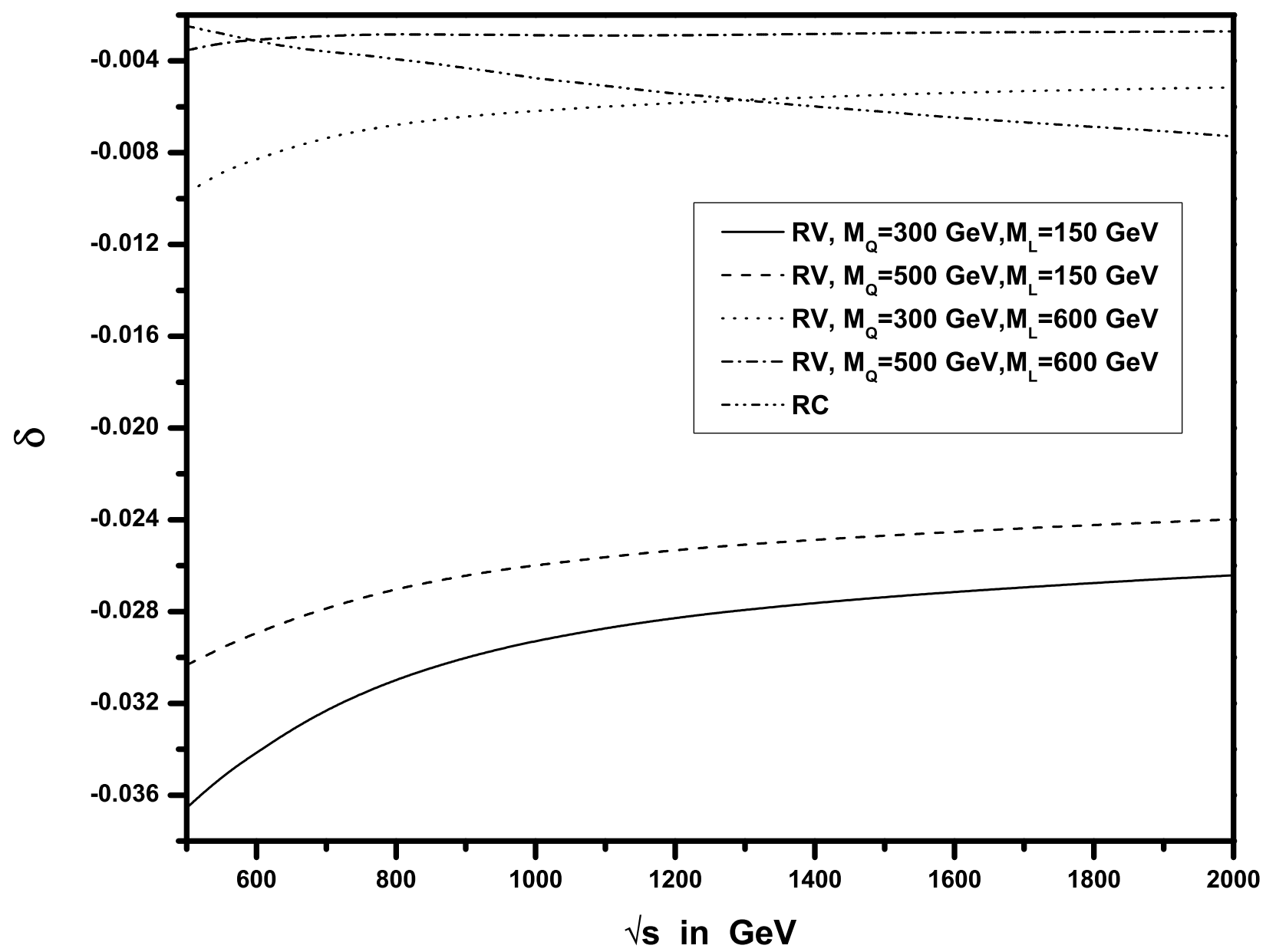

